\title{
Vector-like quarks with non-renormalizable interactions
}

\section{J.C. Criado and M. Pérez-Victoria}

CAFPE and Departamento de Fúsica Teórica y del Cosmos,

Universidad de Granada, Campus de Fuentenueva, E-18071 Granada, Spain

E-mail: jccriadoalamo@ugr.es, mpv@ugr.es

ABSTRACT: We study the impact of the leading non-renormalizable terms in the effective field theory that describes general extensions of the Standard Model with vector-like quarks that can decay into Standard Model particles. Dropping the usual assumption of renormalizability has several phenomenological consequences for the production and decay of the heavy quarks and also for Higgs physics. The most dramatic effects, including those associated with a long lifetime, occur for vector-like quarks with non-standard quantum numbers.

KEYwords: Beyond Standard Model, Effective Field Theories

ArXiv EPrint: 1908.08964 


\section{Contents}

1 Introduction 1

2 Non-renormalizable extensions of the Standard Model with vector-like quarks

3 Mixing

4 Indirect effects

5 Production at the LHC

6 Decay

7 Conclusions

A Representations of Standard Model operators

B Limits on the mass for the case with extra decays

C Approximate equality of the branching ratios to $H q$ and $Z q$

\section{Introduction}

The fermions in the Standard Model (SM) can be classified as leptons and quarks, according to their respective transformations as singlets or triplets under the colour gauge group. Additional spin 1/2 particles with these colour quantum numbers are often considered by theorists and experimentalists in the quest for new physics. This is motivated both by their rich phenomenology and by their frequent occurrence in explicit models. Due to constraints from electroweak precision data and Higgs physics, these new particles cannot acquire their mass only from the Higgs vacuum expectation value (vev). The necessary gauge-invariant mass term requires left-handed and right-handed components, transforming in the same way, not only under the colour group, but also, unlike the SM fermions, under the electroweak gauge group. Dirac fermions with this property are known as vector-like fermions. ${ }^{1}$ Two important properties of vector-like fermions is that all their observable effects decouple when their mass is taken to infinity and that they never give rise to anomalies of the SM gauge group. In this paper we concentrate on vector-like extra quarks. The indirect and direct effects of general vector-like leptons have been analyzed in refs. [1] and [2], respectively.

\footnotetext{
${ }^{1} \mathrm{~A}$ gauge-invariant mass is also possible for Majorana fermions in real representations of the SM gauge group, which can also be considered vector-like fermions.
} 
Vector-like quarks appear in many motivated extensions of the SM, for diverse reasons. In models with additional symmetries, they may complete multiplets that include SM fermions [3-5]. They may also be necessary for the cancellation of the anomalies of an extended gauge group [6]. In models with (partially) composite quarks [7], they emerge effectively as resonances, while in models in extra dimensions, they show up as KaluzaKlein modes when the quarks propagate through the bulk [8]. Vector-like quarks are also used to relax the bounds from precision observables [9] or to avoid strong fine tuning in the Higgs sector $[10,11]$. Here, we will not worry about the origin of the vector-like quarks or the details of the model in which they appear. Instead, we follow a systematic modelindependent approach by studying a general effective field theory that describes the new quarks and their interactions with the SM fields. Our conclusions can be easily translated to specific models.

Most analyses of vector-like quarks so far have assumed renormalizable interactions (we comment on exceptions below). At the renormalizable level, the possible gauge-invariant interactions of the extra quarks in the electroweak symmetric phase are the ones with the gauge bosons, determined by their quantum numbers, and Yukawa interactions involving either two extra quarks or one extra quark and one SM quark. Upon electroweak breaking, the Yukawa couplings give rise to off-diagonal terms in the quark mass matrix, which translate into the mixing of mass eigenstates in the interaction terms with the $Z$ and $W$ bosons and the Higgs (beyond the mixing in the original Yukawas). Many of the observable effects of the new quarks, such as their decay into SM particles, their single production and the induced modifications of the light-quark couplings, are associated to their mixing with the SM quarks, which is suppressed when their gauge-invariant mass is larger than the $Z$ mass [12]. This suppression is stronger for heavy vector-like quarks that are not directly connected by Yukawa couplings to the SM quarks. Therefore, the effects of mixing are sizable only in the presence of vector-like quarks with gauge quantum numbers that allow for such couplings. Assuming that electroweak breaking is mostly triggered by the vev $v$ of one or more Higgs doublets, in agreement with limits on the $\rho$ parameter, there are seven different multiplets of vector-like quarks that carry the appropriate quantum numbers. They are shown in the first seven rows of table 1 . Note that these are the only vector-like quarks that can couple linearly to SM operators in a renormalizable theory. ${ }^{2}$ Vector-like quarks with this property will be called "renormalizable" vector-like quarks (RVLQ), even if they can also have non-renormalizable interactions. Their components have electric charges in the set $\{ \pm 1 / 3, \pm 2 / 3, \pm 4 / 3, \pm 5 / 3\}$. The most general renormalizable extension of the SM with arbitrary combinations of the seven types of RVLQ was explicitly written in ref. [13]. In that work, the leading indirect effects beyond the SM, including flavourchanging neutral currents, right-handed charged currents and a non-unitary CKM matrix, were studied by integrating the heavy quarks out and using the results in ref. [14] for the relevant flavourful part of the SM effective field theory (SMEFT) at dimension six. The loop contributions of these multiplets to oblique parameters have also been calculated in

\footnotetext{
${ }^{2}$ More generally, the only extra fermion fields of spin $1 / 2$ that can couple linearly to SM operators in a renormalizable theory are either colour singlets or colour triplets, that is, either leptons or quarks.
} 
refs. [15-17]. Regarding direct searches, refs. $[18,19]$ provide a comprehensive and detailed guide to the LHC phenomenology of minimal renormalizable extensions of the SM with vector-like quarks that mix dominantly with the third family. Several other works have been devoted to collider searches of RVLQ, see for instance refs. [20-24].

In the present work, we extend this framework by allowing non-renormalizable interactions. This allows us to assess the robustness of the standard limits on vector-like quarks and to explore possible new observable signals. We consider an effective Lagrangian, invariant under the SM gauge symmetry and constructed with the SM fields (including the Higgs doublet) and the vector-like quarks. For simplicity, we will consider simple extensions with only one quark multiplet at a time. The cutoff scale $\Lambda$ of the effective Lagrangian is required to be larger than all the mass scales in the theory, and in particular larger than the gauge invariant mass $M$ of the new quark. All the possible particles not included in the effective Lagrangian, such as additional extra quarks or extra scalars, are assumed to be heavier than $\Lambda$; their effects are then encoded into the Wilson coefficients of the effective theory. As usual, the effective Lagrangian is to be expanded in inverse powers of $\Lambda$. When $\Lambda$ is much higher than the probed energies $E$ and the Higgs vev $v$, all the effects of higherdimensional operators will be suppressed by powers of $E / \Lambda$ and/or $v / \Lambda$ with respect to the effects of the renormalizable ones and will typically give rise to small corrections to the known results. However, some processes may require the presence of higher-dimensional interactions, which will then provide the leading contributions. In particular, this is always the case for quark multiplets that can only couple linearly and gauge invariantly to the SM fermions at the non-renormalizable level. As we will see, the phenomenology of these multiplets can indeed be different from the one of RVLQ.

In fact, relaxing the requirement of renormalizability enlarges the list of vector-like quarks that can mix with the SM ones and, more generally, have linear couplings with SM operators. ${ }^{3}$ The number of different multiplets with this property is finite at each order in $1 / \Lambda$ and increases with the order in this expansion. In this paper, we only study explicitly the leading corrections to renormalizable theories with vector-like quarks. So, we will truncate the effective Lagrangian at order $1 / \Lambda$, that is, we will consider only operators of canonical dimension $n \leq 5$. The quark multiplets that can have linear couplings to this order are collected in table 1. As can be checked there, there are five new multiplets, in addition to the seven RVLQ. The new ones will be called "non-renormalizable" vector-like quarks (NRVLQ). The only gauge-invariant operator that can be built with the SM fields at dimension 5 is the Weinberg operator, which involves only leptons and can thus be ignored in our context. The rest of dimension- 5 operators always contain at least one of the extra quarks in table 1 . In order to simplify the analysis, we will assume that the extra quarks do not couple to the first two SM families. This assumption can easily be dropped, at the price of introducing more free parameters. We study the mixing with the third family of SM quarks and the associated phenomenology, including indirect effects on electroweak and Higgs observables and the production and decay of the new quarks. We will see that

\footnotetext{
${ }^{3}$ Interestingly, non-renormalizable linear interactions of other colour representations, beyond singlets and triplets, are also allowed.
} 
for some multiplets there are new single production mechanisms and new decay channels, which can be sizable in some regions of parameter space. A significant feature of the vectorlike quarks without renormalizable interactions is that their widths are suppressed. For dimensionless couplings of order 1 and a cutoff $\Lambda$ larger than $5 \mathrm{TeV}$, it turns out that their lifetimes are larger than the typical QCD times and thus non-perturbative effects, including hadronization, will take place before decay. For still larger values of $\Lambda$, the NRVLQ, or more precisely the hadrons they form, will be long lived. These quarks would then elude the usual searches, which assume prompt decays, and lead instead to alternative signatures, such as tracks with anomalous ionization, long time of flight or displaced vertices.

Extra quarks with non-renormalizable interactions have been studied before in the context of pseudo-Goldstone composite Higgs models [11, 25-27]. This is a particular subclass of the theories included in our general model-independent framework, with $\Lambda$ identified with the symmetry breaking scale $f$. But in the pseudo-Goldstone scenario, the assumed symmetry breaking pattern allows to easily resum the $1 / f$ expansion. Then, $f$ can be pretty low without loosing predictive power. ${ }^{4}$ The vector-like quarks in those models belong to multiplets of an extended symmetry and, for the popular choices in the literature, decompose under the SM gauge group into a subset of the seven RVLQ representations. Here, we want to follow a model-independent approach, so we do not make any assumptions about the nature of the Higgs, about symmetries beyond the SM ones or about the representations of the quarks (except for the requirement of linear interactions). Another study of non-renormalizable interactions for new quarks, similar in spirit to the one in this paper, was presented in ref. [29]. There, the first three multiplets in table 1, coupled via operators involving the Higgs, were considered. We generalize this work by including all the relevant multiplets and operators at dimension 5. In particular, we consider multiplets without dimension- 4 interactions, which present the most dramatic changes with respect to the usual phenomenology of vector-like quarks. On the other hand, the flavour structure we assume is more restrictive than the one in ref. [29], which allowed for couplings to the light families of SM quarks.

We have implemented in FEYNRules 2.0 [30] the effective theory for each vector-like multiplet in table 1. All the simulations have been performed with MADGRAPH5_AMC@ NLO $[31,32]$ with the UFO files generated with FEYnRuLES.

The paper is organized as follows. In section 2, we introduce the effective theory for vector-like quarks, find the constraints on quantum numbers for linear interactions and write explicitly the general Lagrangian for an arbitrary multiplet with all the operators of dimension up to 5 . We also comment briefly on the possible ultraviolet (UV) origin of the non-renormalizable operators. In section 3, we diagonalize the mass matrices that appear in the Higgs phase for the components with the same electric charges as the SM quarks. Section 4 is devoted to indirect effects of the new quarks and to the corresponding limits from Higgs, electroweak and top data. Production at hadron colliders is discussed in section 5 , while the decay of the new quarks is examined in section 6 . We present our

\footnotetext{
${ }^{4}$ The effective descriptions of these models are valid up to a cutoff higher than $f$, associated to additional resonances or strong coupling. In explicit holographic models, these effects are incorporated and the cutoff can be much higher for many purposes [28].
} 
conclusions in 7. Three appendices are devoted to some important technical results that are used in the main text. In appendix A, we obtain a necessary condition for the linear coupling of new fields to SM operators of arbitrary dimension. In appendix B, we find a simple formula to reinterpret the mass limits provided by the LHC collaborations in the case with additional decay modes. Our method is based on the one in ref. [33]. Finally, in appendix $\mathrm{C}$ we explain why the branching ratios to Higgs and $\mathrm{Z}$ bosons are approximately equal for all multiplets but one.

\section{Non-renormalizable extensions of the Standard Model with vector-like quarks}

Let us consider a general local effective Lagrangian $\mathcal{L}$ describing extensions of the SM with extra vector-like quarks. The effective theory will be valid for energies smaller than a certain cutoff $\Lambda$, which is larger than all the mass scales in the theory, including the mass $M$ of the extra quarks. $\mathcal{L}$ must be invariant under Lorentz and $\mathrm{SU}(3) \times \mathrm{SU}(2) \times \mathrm{U}(1)$ gauge transformations. We assume that the latter is linearly realized. The degrees of freedom that appear in the Lagrangian are the SM fields, including the Higgs doublet, and the new spinors, which transform as triplets under $\mathrm{SU}(3)$. We do not impose renormalizability. Up to this point, the $\mathrm{SU}(2) \times \mathrm{U}(1)$ representation of the new quarks is completely arbitrary, except for the vector-like condition, which requires that the new quarks be grouped in pairs of Weyl spinors belonging to the same representation. Now, let us introduce our main non-trivial restriction: the new quarks are assumed to have linear couplings to the SM fields. In other words, there exist interaction terms involving products of SM fields and a single power of the extra quark field. This assumption is motivated by phenomenology, as explained in the introduction, and has the crucial advantage of restricting the irreducible representations of $\mathrm{SU}(2) \times \mathrm{U}(1)$ to a finite set, when the $1 / \Lambda$ expansion of $\mathcal{L}$ is truncated at any order. Indeed, the isospin and hypercharge of a quark coupling linearly to an SM operator $\mathcal{O}$ is given directly by the isospin and hypercharge of $\mathcal{O}$, which belong to a finite set for a fixed dimension of the operator.

Moreover, we find in appendix A a general constraint over the representation of any Standard Model operator, and thus of any field with a gauge-invariant linear coupling. ${ }^{5}$ In the case of colour triplets, it reads

$$
T+Y+1 / 3 \in \mathbb{Z}
$$

with $T$ the isospin of the $\mathrm{SU}(2)$ representation and $Y$ the hypercharge. It is also true that, given a representation of $\mathrm{SU}(2) \times \mathrm{U}(1)$ satisfying eq. (2.1), there is a product of Standard Model fields that produces this representation. Indeed, consider first the products $\phi^{k}\left(\phi^{*}\right)^{l}$ of the Higgs doublet and its conjugate. They generate all representations with $T+Y \in \mathbb{Z}$. Then, the operators of the form $\phi^{k}\left(\phi^{*}\right)^{l} q$ give all the possibilities satisfying eq. (2.1). So this formula allows to find easily the quark multiplets with linear couplings.

Higher-dimensional multiplets couple linearly to the Standard Model through higherdimensional operators. Therefore, the effects of higher-dimensional multiplets tend to be

\footnotetext{
${ }^{5}$ This condition has been given before, in a different form, in ref. [34].
} 
more suppressed than the lower-dimensional ones. As we have just explained, at each order in inverse powers of the cutoff $\Lambda$, which is given by the dimension of the operators, there is a finite number of multiplets with linear couplings to SM fields. This number increases with the order in $1 / \Lambda$. We focus in the following on the next-to-leading order in this expansion, which is $O(1 / \Lambda)$. Equivalently, we impose a maximum dimension of 5 for the operators in the effective Lagrangian. There are twelve possible multiplets with linear couplings at this order, listed in table 1 . The ones in the first seven rows, called RVLQ in this paper, can have linear interactions of dimension 4 . These are the multiplets that have been studied in the past. For natural values of the couplings, the dimension- 5 operators will generate small corrections to their properties. The remaining five multiplets, which we call NRVLQ, cannot have dimension-4 linear couplings. Therefore, for these multiplets the dimension-5 interactions will give leading-order effects. Let us stress that RVLQ can have non-renormalizable linear interactions and that NRVLQ have renormalizable quadratic interactions with the gauge fields, besides the kinetic and mass terms. Let us also note in passing that, besides the singlet and triplet representations, other irreducible representations of $\mathrm{SU}(3)$ are possible for spin-1/2 particles with dimension-5 linear couplings to the Standard Model. The extra eight possibilities for their representations under $\mathrm{SU}(3) \times \mathrm{SU}(2) \times \mathrm{U}(1)$ are:

$$
(6,1)_{-2 / 3},(6,1)_{1 / 3},(6,2)_{-1 / 6},(8,1)_{1},(8,2)_{1 / 2},(15,1)_{2 / 3},(15,1)_{-1 / 3},(15,2)_{1 / 6} \text {. }
$$

Coming back to extra quarks, the dimension-5 operators containing exactly one vectorlike quark can have one of the following two schematic forms: $\bar{Q} \phi \phi q$ and $\bar{Q} \sigma^{\mu \nu} q F_{\mu \nu}$, where $\phi$ is the Higgs doublet, $q$ and $Q$ represent SM and extra quark multiplets, respectively, and $F_{\mu \nu}$ is the field-strength tensor of a SM gauge field. We do not consider operators with the field content $\bar{Q} \phi q D$, with $D$ a covariant derivative, because they can be eliminated using integration by parts and field redefinitions, up to $O\left(1 / \Lambda^{2}\right)$ corrections. The interactions allowed for each multiplet are presented in table 1 . It is important to note that the interactions of the form $\bar{Q} \phi \phi q$ will typically give physical effects suppressed by powers of $v / \Lambda$, while the effects of interactions of the form $\bar{Q} \sigma^{\mu \nu} q F_{\mu \nu}$ are suppressed by powers of $E / \Lambda$, with $E$ the characteristic energy of the process ( $E \simeq M$ for on-shell extra quarks). In the rest of this paper, we study the theories defined by adding each of the possible multiplets at a time. The dimension- 5 effective Lagrangian for any such theory with one multiplet $Q$ is $\mathcal{L}=\mathcal{L}_{\mathrm{SM}}+\mathcal{L}_{Q}^{\text {free }}+\left(\mathcal{L}_{Q}^{\text {lin }}+\mathcal{L}_{Q}^{\text {quad }}+\right.$ h.c. $)$, with

$$
\begin{aligned}
\mathcal{L}_{Q}^{\text {free }}= & \bar{Q}(i \not D-M) Q \\
-\mathcal{L}_{U}^{\operatorname{lin}}= & \lambda_{i} \bar{U}_{R} \tilde{\phi}^{\dagger} q_{L i}+y_{i}\left(\bar{U}_{L} u_{R i}\right)\left(\phi^{\dagger} \phi\right)+w_{B i} \bar{U}_{L} \sigma^{\mu \nu} u_{R i} B_{\mu \nu}+w_{G i} \bar{U}_{L} \lambda^{A} \sigma^{\mu \nu} u_{R i} G_{\mu \nu}^{A}, \\
-\mathcal{L}_{D}^{\operatorname{lin}}= & \lambda_{i} \bar{D}_{R} \phi^{\dagger} q_{L i}+y_{i}\left(\bar{D}_{L} d_{R i}\right)\left(\phi^{\dagger} \phi\right)+w_{B i} \bar{D}_{L} \sigma^{\mu \nu} d_{R i} B_{\mu \nu} \\
& +w_{G i} \bar{D}_{L} \lambda^{A} \sigma^{\mu \nu} d_{R i} G_{\mu \nu}^{A}, \\
-\mathcal{L}_{Q_{1}}^{\operatorname{lin}}= & \lambda_{u i} \bar{Q}_{1 L} \tilde{\phi} u_{R i}+\lambda_{d i} \bar{Q}_{1 L} \phi d_{R i}+y_{u i}\left(\bar{Q}_{1 R} \tilde{\phi}\right)\left(\tilde{\phi}^{\dagger} q_{L i}\right)+y_{d i}\left(\bar{Q}_{1 R} \phi\right)\left(\phi^{\dagger} q_{L i}\right) \\
& +w_{B i} \bar{Q}_{1 R} \sigma^{\mu \nu} q_{L i} B_{\mu \nu}+w_{W i} \bar{Q}_{1 R} \sigma^{a} \sigma^{\mu \nu} q_{L i} W_{\mu \nu}^{a}+w_{B i} \bar{Q}_{1 R} \lambda^{A} \sigma^{\mu \nu} q_{L i} G_{\mu \nu}^{A}, \\
-\mathcal{L}_{Q_{7}}^{\operatorname{lin}}= & \lambda_{i} \bar{Q}_{7 L} \phi u_{R i}+y_{i}\left(\bar{Q}_{7 R} \phi\right)\left(\tilde{\phi}^{\dagger} q_{L i}\right),
\end{aligned}
$$




$$
\begin{aligned}
-\mathcal{L}_{Q_{5}}^{\operatorname{lin}}= & \lambda_{i} \bar{Q}_{5 L} \tilde{\phi} d_{R i}+y_{i}\left(\bar{Q}_{5 R} \tilde{\phi}\right)\left(\phi^{\dagger} q_{L i}\right) \\
-\mathcal{L}_{T_{1}}^{\operatorname{lin}}= & \lambda_{i} \bar{T}_{1 R}^{a} \phi^{\dagger} \sigma^{a} q_{L i}+y_{u i} \bar{T}_{1 L}^{a} u_{R i} \phi^{\dagger} \sigma^{a} \tilde{\phi}+y_{d i} \bar{T}_{1 L}^{a} d_{R i} \phi^{\dagger} \sigma^{a} \phi \\
& +w_{i} \bar{T}_{1 L}^{a} \sigma^{\mu \nu} d_{R i} W_{\mu \nu}^{a} \\
-\mathcal{L}_{T_{2}}^{\operatorname{lin}}= & \lambda_{i} \bar{T}_{2 R}^{a} \tilde{\phi}^{\dagger} \sigma^{a} q_{L i}+y_{u i} \bar{T}_{2 L}^{a} u_{R i} \phi^{\dagger} \sigma^{a} \phi+y_{d i} \bar{T}_{2 L}^{a} d_{R i} \tilde{\phi}^{\dagger} \sigma^{a} \phi \\
& +w_{i} \bar{T}_{2 L}^{a} \sigma^{\mu \nu} u_{R i} W_{\mu \nu}^{a} \\
-\mathcal{L}_{T_{4}}^{\operatorname{lin}}= & y_{i} \bar{T}_{4 L}^{a} d_{R i} \phi^{\dagger} \sigma^{a} \tilde{\phi} \\
-\mathcal{L}_{T_{5}}^{\operatorname{lin}}= & y_{i} \bar{T}_{5 L}^{a} u_{R i} \tilde{\phi}^{\dagger} \sigma^{a} \phi \\
-\mathcal{L}_{F_{1}}^{\operatorname{lin}}= & y_{i} \bar{F}_{1 R}^{a} C_{b c}^{a} q_{L i c} \phi^{\dagger} \sigma^{b} \phi+w_{i} \bar{F}_{1 R}^{a} C_{b c}^{a} \sigma^{\mu \nu} q_{L i c} W_{\mu \nu}^{b} \\
-\mathcal{L}_{F_{5}}^{\operatorname{lin}}= & y_{i} \bar{F}_{5 R}^{a} C_{b c}^{a} q_{L i c} \phi^{\dagger} \sigma^{b} \tilde{\phi} \\
-\mathcal{L}_{F_{7}}^{\operatorname{lin}}= & y_{i} \bar{F}_{7 R}^{a} C_{b c}^{a} q_{L i c} \tilde{\phi}^{\dagger} \sigma^{b} \phi \\
-\mathcal{L}_{Q}^{\text {quad }}= & W_{B}\left(\bar{Q}_{L} \sigma^{\mu \nu} Q_{R}\right) B_{\mu \nu}+W_{W}\left(\bar{Q}_{L} \sigma^{\mu \nu} \mathbf{T}_{Q}^{a} Q_{R}\right) W_{\mu \nu}^{a}+W_{G}\left(\bar{Q}_{L} \sigma^{\mu \nu} \mathbf{t}_{Q}^{A} Q_{R}\right) G_{\mu \nu}^{A} \\
& +Y_{1}\left(\bar{Q}_{L} Q_{R}\right)\left(\phi^{\dagger} \phi\right)+Y_{2}\left(\bar{Q}_{L} \mathbf{T}_{Q}^{a} Q_{R}\right)\left(\phi^{\dagger} \sigma^{a} \phi\right)
\end{aligned}
$$

where $\lambda^{A}$ are the Gell-Mann matrices, $\sigma^{a}$ are the Pauli matrices, $\mathbf{T}_{Q}^{A}\left(\mathbf{t}_{Q}^{a}\right)$ are the generators of $\mathrm{SU}(2)(\mathrm{SU}(3))$ in the representation of $Q$, and the matrices $C^{a}$ are defined by

$$
\begin{array}{rlrl}
C^{3 / 2} & =\frac{1}{\sqrt{2}}\left(\begin{array}{rr}
1 & 0 \\
-i & 0 \\
0 & 0
\end{array}\right), & C^{1 / 2}=\frac{1}{\sqrt{6}}\left(\begin{array}{rr}
0 & 1 \\
0 & -i \\
-2 & 0
\end{array}\right), \\
C^{-1 / 2}=-\frac{1}{\sqrt{6}}\left(\begin{array}{cc}
1 & 0 \\
i & 0 \\
0 & 2
\end{array}\right), & C^{-3 / 2}=-\frac{1}{\sqrt{2}}\left(\begin{array}{cc}
0 & 1 \\
0 & i \\
0 & 0
\end{array}\right) .
\end{array}
$$

These matrices connect the quadruplet representation of SU(2) with the doublet and triplet representations. The index $i$ indicates the SM fermion family and $W_{W}=Y_{2}=0$ for singlets. We have used the following notation for coefficients of operators that are linear in $Q: \lambda_{i}$ is the coefficient of $\bar{Q} q_{i} \phi, y_{i}$ is for $\bar{Q} q_{i} \phi \phi$, and $w_{i}$ is for $\bar{Q} \sigma^{\mu \nu} q_{i} F_{\mu \nu}$. When there is more than one possibility, the corresponding coupling constants are differentiated by an additional subindex, which indicates the SM field that unambiguously determines the operator. Observe that we include all the gauge-invariant operators of dimension equal to or smaller than 5 that can be constructed with the field content of the theory. The condition of linear couplings is used to select the representations of the vector-like quarks, but not to restrict their interactions in the effective theory. Note also that the $\lambda_{i}$ parameters are dimensionless, whereas $y_{i}, w_{i}, Y$ and $W$ have dimensions of inverse energy and are expected to be of order $\Lambda^{-1}$. We will consider in this paper only couplings to the third family of SM quarks. This choice is made to reduce the dimensionality of the parameter space and to automatically satisfy the most stringent flavour limits. It is also motivated by theoretical ideas in different models. This means that $\lambda_{i}, y_{i}$ and $w_{i}$ are taken to be vanishing for $i=1,2$. Accordingly, we simplify the name of the non-vanishing couplings in 


\begin{tabular}{|ccccc|}
\hline Name & Irrep & $\bar{Q} \phi q$ & $\bar{Q} \phi \phi q$ & $\bar{Q} \sigma^{\mu \nu} q F_{\mu \nu}$ \\
\hline$U$ & $1_{2 / 3}$ & $\checkmark$ & $\checkmark$ & $\checkmark$ \\
$D$ & $1_{-1 / 3}$ & $\checkmark$ & $\checkmark$ & $\checkmark$ \\
$Q_{1}$ & $2_{1 / 6}$ & $\checkmark$ & $\checkmark$ & $\checkmark$ \\
$Q_{5}$ & $2_{-5 / 6}$ & $\checkmark$ & $\checkmark$ & $\boldsymbol{x}$ \\
$Q_{7}$ & $2_{7 / 6}$ & $\checkmark$ & $\checkmark$ & $\boldsymbol{x}$ \\
$T_{1}$ & $3_{-1 / 3}$ & $\checkmark$ & $\checkmark$ & $\checkmark$ \\
$T_{2}$ & $3_{2 / 3}$ & $\checkmark$ & $\checkmark$ & $\checkmark$ \\
$T_{4}$ & $3_{-4 / 3}$ & $\boldsymbol{x}$ & $\checkmark$ & $\boldsymbol{x}$ \\
$T_{5}$ & $3_{5 / 3}$ & $\boldsymbol{x}$ & $\checkmark$ & $\boldsymbol{x}$ \\
$F_{1}$ & $4_{1 / 6}$ & $\boldsymbol{x}$ & $\checkmark$ & $\checkmark$ \\
$F_{5}$ & $4_{-5 / 6}$ & $\boldsymbol{x}$ & $\checkmark$ & $\boldsymbol{x}$ \\
$F_{7}$ & $4_{7 / 6}$ & $\boldsymbol{x}$ & $\boldsymbol{\checkmark}$ & $\boldsymbol{x}$ \\
\hline
\end{tabular}

Table 1. Irreps $(2 T+1)_{Y}$ under $\mathrm{SU}(2)_{L} \times \mathrm{U}(1)_{Y}$ and linear interactions of new quarks with dimension-5 linear couplings. The subscript in the name of each multiplet is the absolute value of the numerator of its hypercharge, when written as an irreducible fraction. An explicit formula for this integer number is $|2+4 \widetilde{T}+3(Y-2 / 3) /(1-\widetilde{T})|$ where $\widetilde{T}=T(\bmod 1)$.

the following way:

$$
\begin{aligned}
& \lambda=\lambda_{3} ; \quad \lambda_{t}=\lambda_{u 3} ; \quad \lambda_{b}=\lambda_{d 3} ; \\
& y=y_{3} ; \quad y_{t}=y_{u 3} ; \quad y_{b}=y_{d 3} ; \\
& w=w_{3} ; \quad w_{B}=w_{B 3} ; \quad w_{W}=w_{W 3} ; \quad w_{G}=w_{G 3} .
\end{aligned}
$$

Let us briefly comment on possible ultraviolet completions that can give rise to the dimension 5 operators at low energies. The Yukawa-like operators $\bar{Q} q \phi \phi$, of dimension 5 , can be generated at the tree level in a completion with one additional field: either a colour-neutral scalar $\mathbf{S}$, with interactions $\mathbf{S} \bar{Q} q$ and $\mathbf{S} \phi \phi$ or an additional quark $\mathbf{Q}$, with interactions $\mathbf{Q} \phi Q$ and $\mathbf{Q} \phi q$. The mass of the extra particle, which is assumed to be larger than $M$, sets the cutoff scale $\Lambda$ of the effective theory $\mathcal{L}$. The Feynman diagrams that contribute to the dimension-5 Yukawas are shown in figure 1 . The quantum numbers of the extra field must allow for the gauge-invariant vertices in the diagrams. This means that the heavy scalar $\mathbf{S}$ belongs to one of the representations $1_{0}, 3_{0}$ and $3_{1}$ of $\mathrm{SU}(2) \times \mathrm{U}(1)$, while the heavy quark $\mathbf{Q}$ belongs to one of the representations in the first seven rows of table 1 , so it is also a RVLQ (but assumed to be heavier than the ones in the effective Lagrangian). The operators of the form $\bar{Q} \sigma_{\mu \nu} q F^{\mu \nu}$, on the other hand, cannot be generated at treelevel in a renormalizable ultraviolet theory. In figure 2 we show a one-loop diagram that contributes to these effective operators in a theory with an extra scalar multiple $\mathbf{S}$, which must be either a singlet or a triplet of $\mathrm{SU}(2)$, and a singlet or an octet of $\mathrm{SU}(3)$. That is, there are 4 possibilities: $(1,1)_{0},(1,3)_{0},(8,1)_{0}$ and $(8,3)_{0}$. The coefficients $w$ of these "magnetic" operators are thus naturally suppressed by a loop factor in weakly coupled completions. In addition, because a quark mass insertion $m_{Q}$ is needed for the chiralities 

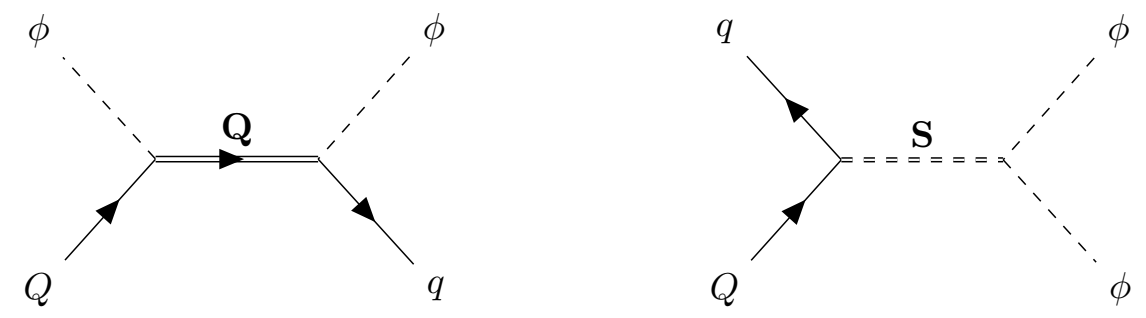

Figure 1. Tree-level diagrams that generate the $\bar{Q} q \phi \phi$ operator in UV completions of $\mathcal{L}$ with additional extra quarks (left) and additional scalars (right).

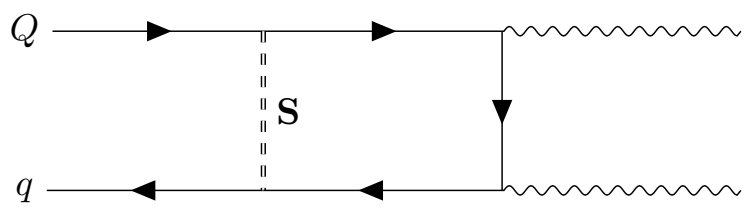

Figure 2. A one-loop diagram that generates the $\bar{Q} \sigma_{\mu \nu} q F^{\mu \nu}$ operator in a UV completion of $\mathcal{L}$ with new scalars.

of the external lines to match those of the effective operator, the suppression with the UV scale $m_{S}$ is not $1 / m_{S}$ as expected from the effective theory power counting, but $m_{Q} / m_{S}^{2}$. An explicit model with a $U$ vector-like quark and a scalar singlet has been studied in [35].

\section{$3 \quad$ Mixing}

The multiplets in table 1 can be decomposed into component fields with well-defined electric charge:

$$
\begin{aligned}
Q_{1}=\left(\begin{array}{c}
T^{0} \\
B^{0}
\end{array}\right), & Q_{5}=\left(\begin{array}{c}
B^{0} \\
Y
\end{array}\right), & Q_{7}=\left(\begin{array}{c}
X \\
T^{0}
\end{array}\right) \\
T_{1}=\left(\begin{array}{c}
T^{0} \\
B^{0} \\
Y
\end{array}\right), & T_{2}=\left(\begin{array}{c}
X \\
T^{0} \\
B^{0}
\end{array}\right), & T_{4}=\left(\begin{array}{c}
B^{0} \\
Y \\
Y^{\prime}
\end{array}\right), \quad T_{5}=\left(\begin{array}{c}
X^{\prime} \\
X \\
T^{0}
\end{array}\right) \\
F_{1}=\left(\begin{array}{c}
X \\
T^{0} \\
B^{0} \\
Y
\end{array}\right), & F_{5}=\left(\begin{array}{c}
X^{\prime} \\
B^{0} \\
Y \\
Y^{\prime}
\end{array}\right), & F_{7}=\left(\begin{array}{c}
X \\
T^{0} \\
B^{0}
\end{array}\right)
\end{aligned}
$$

The components are denoted by symbols in the set $\left\{X^{\prime}, X, T^{0}, B^{0}, Y, Y^{\prime}\right\}$, with electric charges given by

$$
\begin{aligned}
Q\left(X^{\prime}\right) & =8 / 3, & Q\left(B^{0}\right) & =-1 / 3, \\
Q(X) & =5 / 3, & Q(Y) & =-4 / 3, \\
Q\left(T^{0}\right) & =2 / 3, & Q\left(Y^{\prime}\right) & =-7 / 3 .
\end{aligned}
$$


Upon electroweak breaking, the fields $T^{0}\left(B^{0}\right)$ will mix, in general, with all the Standard Model up-type (down-type) quarks. However, with our flavour restriction and neglecting the tiny off-diagonal CKM elements of the third family, the new quarks mix only with the top and bottom quarks. The relevant mass terms have the form

$$
\begin{aligned}
\mathcal{L}_{\text {mass }}= & -\left(\begin{array}{ll}
\bar{t}_{L}^{0} & \bar{T}_{L}^{0}
\end{array}\right)\left(\begin{array}{ll}
m_{11}^{t} & m_{12}^{t} \\
m_{21}^{t} & m_{22}^{t}
\end{array}\right)\left(\begin{array}{c}
t_{R}^{0} \\
T_{R}^{0}
\end{array}\right) \\
& -\left(\begin{array}{ll}
\bar{b}_{L}^{0} & \bar{B}_{L}^{0}
\end{array}\right)\left(\begin{array}{ll}
m_{11}^{b} & m_{12}^{b} \\
m_{21}^{b} & m_{22}^{b}
\end{array}\right)\left(\begin{array}{c}
b_{R}^{0} \\
B_{R}^{0}
\end{array}\right)+\text { h.c. }
\end{aligned}
$$

with the superindex 0 emphasizing that the fields are weak eigenstates, i.e. the components of the gauge-covariant multiplets. ${ }^{6}$ The elements of the diagonal of each of the mass matrices are $m_{11} \sim v$, which arises from the Standard Model Yukawa coupling $\bar{q} \phi q$, and $m_{22} \simeq M$. For RVLQ, one of the off-diagonal elements, $m_{i j} \sim v$, comes from the operator $\bar{Q} \phi q$, and the other one, $m_{j i} \sim y v^{2}$, comes from $\bar{Q} \phi \phi q$. For NRVLQ, only one of the off-diagonal elements, $m_{i j} \sim y v^{2}$, is non-zero. The precise values of the entries of the mass matrices are given in table 2 . The mixing angles that relate weak and mass eigenstates are obtained by diagonalizing the corresponding mass matrices:

$$
\begin{aligned}
\left(\begin{array}{c}
t_{L, R} \\
T_{L, R}
\end{array}\right) & =\left(\begin{array}{cc}
c_{L, R}^{t} & -e^{i \phi_{t}} s_{L, R}^{t} \\
e^{-i \phi_{t}} s_{L, R}^{u} & c_{L, R}^{t}
\end{array}\right)\left(\begin{array}{c}
t_{L, R}^{0} \\
T_{L, R}^{0}
\end{array}\right), \\
\left(\begin{array}{c}
b_{L, R} \\
B_{L, R}
\end{array}\right) & =\left(\begin{array}{cc}
c_{L, R}^{b} & -e^{i \phi_{b}} s_{L, R}^{b} \\
e^{-i \phi_{b}} s_{L, R}^{b} & c_{L, R}^{b}
\end{array}\right)\left(\begin{array}{c}
b_{L, R}^{0} \\
B_{L, R}^{0}
\end{array}\right),
\end{aligned}
$$

where $t, T, b$ and $B$ are the mass eigenstates, $c_{L, R}^{t, b}:=\cos \theta_{L, R}^{t, b}$ and $s_{L, R}^{t, b}:=\sin \theta_{L, R}^{t, b}$, with $\theta_{L, R}^{t, b}$ the mixing angle. In what follows, we take $\phi_{t}=\phi_{b}=0$, since non-trivial phases $\phi_{t, b}$ can be ignored for the observables discussed here. The explicit expressions for the mixing angles in terms of $m_{i j}^{t, b}$ are (see also ref. [36])

$$
\begin{aligned}
\tan 2 \theta_{L}^{t, b} & =\frac{2\left|m_{11}^{t, b}\left(m_{21}^{t, b}\right)^{*}+m_{12}^{t, b}\left(m_{22}^{t, b}\right)^{*}\right|}{\left|m_{11}^{t, b}\right|^{2}-\left|m_{12}^{t, b}\right|^{2}-\left|m_{21}^{t, b}\right|^{2}+\left|m_{22}^{t, b}\right|^{2}}, \\
\tan 2 \theta_{R}^{t, b} & =\frac{2\left|\left(m_{11}^{t, b}\right)^{*} m_{12}^{t, b}+\left(m_{21}^{t, b}\right)^{*} m_{22}^{t, b}\right|}{\left|m_{11}^{t, b}\right|^{2}-\left|m_{12}^{t, b}\right|^{2}-\left|m_{21}^{t, b}\right|^{2}+\left|m_{22}^{t, b}\right|^{2}} .
\end{aligned}
$$

From these formulas and the scale dependence of each entry it can then be seen that, for $M \gg v$ (in agreement with experimental limits, see below), the mixing angles are suppressed by $v / M$, at least. Furthermore, $\theta_{L} \gg \theta_{R}$ if $\left|m_{12}\right| \gg\left|m_{21}\right|$, and viceversa. For natural values of the couplings and $\Lambda>1 \mathrm{TeV}$, one of the off-diagonal couplings is indeed much larger than the other, so the off-diagonal couplings involving heavy and light

\footnotetext{
${ }^{6}$ Note that we use the symbol $t_{R}^{0}$ for the right-handed SM weak eigenstate of electric charge $3 / 2(-1 / 3)$, which is in fact the unique component of the SM iso-singlet $u_{R 3}\left(d_{R 3}\right)$ of hypercharge $3 / 2(-1 / 3)$. Of course, $t_{L}^{0}\left(b_{L}^{0}\right)$ are the upper and lower components of the SM iso-doublet $q_{L 3}$.
} 


\begin{tabular}{|ccccccc|}
\hline & $m_{12}^{t}$ & $m_{21}^{t}$ & $m_{22}^{t}$ & $m_{12}^{b}$ & $m_{21}^{b}$ & $m_{22}^{b}$ \\
\hline$U$ & $\frac{\lambda^{*} v}{\sqrt{2}}$ & $\frac{y v^{2}}{2}$ & $\hat{M}$ & - & - & - \\
$D$ & - & - & - & $\frac{\lambda^{*} v}{\sqrt{2}}$ & $\frac{y v^{2}}{2}$ & $\hat{M}$ \\
\hline$Q_{1}$ & $\frac{\left(y_{u}\right)^{*} v^{2}}{2}$ & $\frac{\lambda_{u} v}{\sqrt{2}}$ & $\hat{M}-\frac{Y_{2} v^{2}}{4}$ & $\frac{\left(y_{d}\right)^{*} v^{2}}{2}$ & $\frac{\lambda_{d} v}{\sqrt{2}}$ & $\hat{M}+\frac{Y_{2} v^{2}}{4}$ \\
$Q_{5}$ & - & - & - & $\frac{y^{*} v^{2}}{2}$ & $\frac{\lambda v}{\sqrt{2}}$ & $\hat{M}-\frac{Y_{2} v^{2}}{4}$ \\
$Q_{7}$ & $\frac{y^{*} v^{2}}{2}$ & $\frac{\lambda v}{\sqrt{2}}$ & $\hat{M}+\frac{Y_{2} v^{2}}{4}$ & - & - & - \\
\hline$T_{1}$ & $\lambda^{*} v$ & $\frac{y_{u} v^{2}}{\sqrt{2}}$ & $\hat{M}-\frac{Y_{2} v^{2}}{2}$ & $-\frac{\lambda^{*} v}{\sqrt{2}}$ & $-\frac{y_{d} v^{2}}{2}$ & $\hat{M}$ \\
$T_{2}$ & $\frac{\lambda^{*} v}{\sqrt{2}}$ & $-\frac{y u v^{2}}{2}$ & $\hat{M}$ & $\lambda^{*} v$ & $\frac{y_{d} v^{2}}{\sqrt{2}}$ & $\hat{M}+\frac{Y_{2} v^{2}}{2}$ \\
$T_{4}$ & - & - & - & 0 & $\frac{y v^{2}}{\sqrt{2}}$ & $\hat{M}-\frac{Y_{2} v^{2}}{2}$ \\
$T_{5}$ & 0 & $\frac{y v^{2}}{\sqrt{2}}$ & $\hat{M}+\frac{Y_{2} v^{2}}{2}$ & - & - & - \\
\hline$F_{1}$ & $-\frac{y^{*} v^{2}}{\sqrt{6}}$ & 0 & $\hat{M}-\frac{Y_{2} v^{2}}{4}$ & $-\frac{y^{*} v^{2}}{\sqrt{6}}$ & 0 & $\hat{M}+\frac{Y_{2} v^{2}}{4}$ \\
$F_{5}$ & $\frac{y^{*} v^{2}}{\sqrt{2}}$ & 0 & $\hat{M}-\frac{3 Y_{2} v^{2}}{4}$ & $\frac{y^{*} v^{2}}{\sqrt{6}}$ & 0 & $\hat{M}-\frac{Y_{2} v^{2}}{4}$ \\
$F_{7}$ & $-\frac{y^{*} v^{2}}{\sqrt{6}}$ & 0 & $\hat{M}+\frac{Y_{2} v^{2}}{4}$ & $-\frac{y^{*} v^{2}}{\sqrt{2}}$ & 0 & $\hat{M}+\frac{3 Y_{2} v^{2}}{4}$ \\
\hline
\end{tabular}

Table 2. Mass matrix elements. We use the notation $\hat{M}=M+Y_{1} v^{2} / 2$. The 11 component is always just the Standard Model contribution: $m_{11}^{t, b}=\lambda_{\mathrm{SM}}^{t, b} v / \sqrt{2}$.

quark eigenstates will be mostly chiral (especially in the $b$ sector). For RVLQ, the dominant mixing angle is $\theta_{L}$ for even isospin and $\theta_{R}$ for those with odd isospin. For NRVLQ, instead, the dominant mixing angle is $\theta_{R}$ for even isospin and $\theta_{L}$ for odd isospin. Note, however, that for some RVLQ the limits from electroweak precision tests are quite strict [19]. For these multiplets, the off-diagonal entries might be comparable and then the interactions involving both chiralities would be relevant.

\section{Indirect effects}

In this section, we discuss the indirect effects of heavy quarks in low-energy physics, Higgs physics and top physics, which are summarized in table 3. NRVLQ typically generate smaller contributions than RVLQ, as any insertion of a dimension-5 operator introduces a suppression of $1 / \Lambda$. For the same reason, the effects of the dimension-5 interactions of RVLQ will naturally be small corrections to the ones coming only from dimension-4 interactions, when they are present.

Integrating out the RVLQ at tree level gives contributions to dimension- 6 operators in the SMEFT. The low-energy effective Lagrangian, which can be read from ref. [37], is presented in table 4, with the corresponding effective operators defined in table 5. Observe that the dimension-6 terms without extra quarks in the effective theory $\mathcal{L}$, which we are not writing here, will give additional contributions to the corresponding dimension- 6 operators in the SMEFT. However, these contributions will be suppressed by $M^{2} / \Lambda^{2}$ or $M / \Lambda$ relative 


\begin{tabular}{|c|c|c|c|}
\hline & Observable & Coupling & Loop order \\
\hline \multirow{3}{*}{ EWPO } & $S$ and $T$ parameters & $\lambda_{(t)}, y_{(t)}$ & one loop \\
\hline & \multirow{2}{*}{$Z \rightarrow b b$} & $\lambda_{(t)}, y_{(t)}$ & one loop \\
\hline & & $\lambda_{(b)}, y_{(b)}$ & tree level \\
\hline \multirow{4}{*}{ Higgs } & $H \rightarrow b b$ & $\lambda_{(b)}, \lambda_{(b)} y_{(b)}$ & tree level \\
\hline & $t t H$ production & $\lambda$ & tree level \\
\hline & $g g \rightarrow H, H \rightarrow g g$ & $\lambda_{(t)}, Y_{1}$ & one loop \\
\hline & double Higgs production & $\lambda_{(t)}, Y_{1}$ & one loop \\
\hline \multirow{3}{*}{ Top } & top single production & $\lambda_{(t)} w_{W}$ & tree level \\
\hline & top pair production & $\lambda_{(t)}, \lambda_{(t)} w_{B}$ & tree level \\
\hline & $t t \gamma$ and $t t Z$ production & $\lambda_{(t)}, \lambda_{(t)} w_{B}, \lambda_{(t)} w_{W}$ & tree level \\
\hline low-energy CP & electron/neutron EDM & $\lambda_{(t)}, \lambda_{(t)} \lambda_{(b)}, \lambda_{(t)} w_{F}$ & two loops \\
\hline
\end{tabular}

Table 3. Summary of indirect effects of heavy quarks. The subindex $(q)$ means that only the couplings to the Standard Model quark $q$ should be taken. The dependence on products of couplings may involve complex conjugation of some of them.

to the ones from integrating out the RVLQ. Still, they might be relevant for $M / \Lambda$ not small, depending on the values of the couplings. ${ }^{7}$ Here we assume that even in this case they do not cancel against the ones in table 4 .

On the other hand, the NRVLQ do not contribute at tree level to the dimension-6 SMEFT. Therefore, their indirect effects are small. Their leading tree-level contributions of NRVLQ have at least dimension 8 and will not be written explicitly.

Electroweak precision observables. Electroweak precision observables set the strongest limits on the Yukawa couplings of each multiplet. In the mass-eigenstate basis, the mixing between the Standard Model $b$ quark and the $B$ component of a given multiplet induces a modification of the $Z b b$ coupling, which affects the $R_{b}, A_{F B}^{b}, A_{b}$ and $R_{c}$ observables at tree level. $t-T$ mixing changes the $Z t t$ coupling. Insertions of this modified interaction in diagrams with loops of the top quark also generate corrections to these observables, as well as to the $S$ and $T$ parameters.

For the renormalizable multiplets, the origin of these effects can be easily identified in the unbroken phase. They come from tree-level and one-loop diagrams containing the $\mathcal{O}_{\phi q}$-type operators generated by tree level matching. Notice that the non-renormalizable multiplets will also have contributions to these observables, but to obtain them one needs to keep dimension- 8 operators, which indicates that their effects will be smaller.

In ref. [19], the limits on the mixing angles from electroweak precision observables were computed, assuming renormalizability. The corrections from dimension- 5 interactions can

\footnotetext{
${ }^{7}$ This is nothing but a more precise formulation of the usual caution one should exert in general with indirect bounds. Remember that we are considering the case in which only the quarks in one vector-like multiplet are lighter than $\Lambda$. In the presence of other light extra particles (including vector-like quarks) the indirect bounds would need to be reevaluated.
} 


\begin{tabular}{|c|c|c|}
\hline & $\mathcal{L}_{\mathrm{nh}}$ & $\mathcal{L}_{\mathrm{h}}$ \\
\hline$U$ & $\frac{\lambda\left(w_{B}\right)^{*}}{M} \mathcal{O}_{t B}+\frac{\lambda\left(w_{G}\right)^{*}}{M} \mathcal{O}_{t G}+\left(\frac{\left(\lambda_{S M}^{t}\right)^{*}|\lambda|^{2}}{2 M^{2}}+\frac{\lambda^{*} y}{M}\right) \mathcal{O}_{t \phi}$ & $\frac{|\lambda|^{2}}{4 M^{2}} \mathcal{O}_{\phi q}^{(1)}-\frac{|\lambda|^{2}}{4 M^{2}} \mathcal{O}_{\phi q}^{(3)}$ \\
\hline$D$ & $\frac{\lambda\left(w_{B}\right)^{*}}{M} \mathcal{O}_{b B}+\frac{\lambda\left(w_{G}\right)^{*}}{M} \mathcal{O}_{b G}+\left(\frac{\left(\lambda_{\mathrm{SM}}^{b}\right)^{*}|\lambda|^{2}}{2 M^{2}}+\frac{\lambda^{*} y}{M}\right) \mathcal{O}_{b \phi}$ & $-\frac{|\lambda|^{2}}{4 M^{2}} \mathcal{O}_{\phi q}^{(1)}-\frac{|\lambda|^{2}}{4 M^{2}} \mathcal{O}_{\phi q}^{(3)}$ \\
\hline & $\begin{array}{c}\frac{\lambda_{t}\left(w_{B}\right)^{*}}{M} \mathcal{O}_{t B}+\frac{\lambda_{t}\left(w_{W}\right)^{*}}{M} \mathcal{O}_{t W}+\frac{\lambda_{t}\left(w_{G}\right)^{*}}{M} \mathcal{O}_{t G} \\
+\frac{\lambda_{b}\left(w_{B}\right)^{*}}{M} \mathcal{O}_{b B}+\frac{\lambda_{b}\left(w_{W}\right)^{*}}{M} \mathcal{O}_{b W}+\frac{\lambda_{b}\left(w_{G}\right)^{*}}{M} \mathcal{O}_{b G} \\
+\left(\frac{\left(\lambda_{S M}^{t}\right)^{*}\left|\lambda_{t}\right|^{2}}{2 M^{2}}+\frac{\lambda_{t}\left(y_{t}\right)^{*}}{M}\right) \mathcal{O}_{t \phi} \\
+\left(\frac{\left(y_{S M}^{b}\right)^{*}\left|\lambda_{b}\right|^{2}}{2 M^{2}}+\frac{\lambda_{b}\left(y_{b}\right)^{*}}{M}\right) \mathcal{O}_{b \phi}\end{array}$ & $\begin{array}{l}-\frac{\left|\lambda_{t}\right|^{2}}{2 M^{2}} \mathcal{O}_{\phi t}+\frac{\left|\lambda_{b}\right|^{2}}{2 M^{2}} \mathcal{O}_{\phi b} \\
\quad+\frac{\lambda_{b}\left(\lambda_{t}\right)^{*}}{M^{2}} \mathcal{O}_{\phi t b}\end{array}$ \\
\hline$Q_{5}$ & $\left(\frac{\left(\lambda_{S M}^{b}\right)^{*}|\lambda|^{2}}{2 M^{2}}+\frac{\lambda y^{*}}{M}\right) \mathcal{O}_{b \phi}$ & $-\frac{|\lambda|^{2}}{2 M^{2}} \mathcal{O}_{\phi b}$ \\
\hline$Q_{7}$ & $\left(\frac{\left(\lambda_{\mathrm{SM}}^{t}\right)^{*}|\lambda|^{2}}{2 M^{2}}+\frac{\lambda y^{*}}{M}\right) \mathcal{O}_{t \phi}$ & $\frac{|\lambda|^{2}}{2 M^{2}} \mathcal{O}_{\phi t}$ \\
\hline & $\begin{array}{l}\frac{\lambda\left(w_{W}\right)^{*}}{M} \mathcal{O}_{b W}+\left(\frac{\left(y_{\mathrm{SM}}^{t}\right)^{*}|\lambda|^{2}}{4 M^{2}}+\frac{\lambda^{*} y_{t}}{M}\right) \mathcal{O}_{t \phi} \\
\quad+\left(\frac{\left(y_{\mathrm{SM}}^{b}\right)^{*}|\lambda|^{2}}{8 M^{2}}+\frac{\lambda^{*} y_{b}}{2 M}\right) \mathcal{O}_{b \phi}\end{array}$ & $-\frac{3|\lambda|^{2}}{16 M^{2}} \mathcal{O}_{\phi q}^{(1)}+\frac{|\lambda|^{2}}{16 M^{2}} \mathcal{O}_{\phi q}^{(3)}$ \\
\hline$T_{2}$ & $\begin{array}{c}\frac{\lambda\left(w_{W}\right)^{*}}{M} \mathcal{O}_{t W}+\left(\frac{\left(y_{\mathrm{SM}}^{t}\right)^{*}|\lambda|^{2}}{8 M^{2}}-\frac{\lambda^{*} y_{b}}{2 M}\right) \mathcal{O}_{t \phi} \\
+\left(\frac{\left(y_{\mathrm{SM}}^{b}\right)^{*}|\lambda|^{2}}{4 M^{2}}+\frac{\lambda^{*} y_{b}}{M}\right) \mathcal{O}_{b \phi}\end{array}$ & $\frac{3|\lambda|^{2}}{16 M^{2}} \mathcal{O}_{\phi q}^{(1)}+\frac{|\lambda|^{2}}{16 M^{2}} \mathcal{O}_{\phi q}^{(3)}$ \\
\hline
\end{tabular}

Table 4. Dimension-6 effective Lagrangian generated by tree-level matching of the effective theory with each multiplet to the SMEFT. The contributions to Hermitian and non-Hermitian operators are separated in $\mathcal{L}_{\mathrm{h}}$ and $\mathcal{L}_{\text {nh }}$. The complete effective Lagrangian is $\mathcal{L}_{\mathrm{h}}+\left(\mathcal{L}_{\text {nh }}+\right.$ h.c. $)$. The definitions of the operators $\mathcal{O}_{i}$ are given in table 5 .

\begin{tabular}{|cccc|}
\hline Name & Operator & Name & Operator \\
\hline $\mathcal{O}_{b \phi}$ & $\left(\phi^{\dagger} \phi\right)\left(\bar{q}_{L 3} \phi d_{R 3}\right)$ & $\mathcal{O}_{t \phi}$ & $\left(\phi^{\dagger} \phi\right)\left(\bar{q}_{L 3} \phi u_{R 3}\right)$ \\
$\mathcal{O}_{\phi b}$ & $\left(\phi^{\dagger} i \stackrel{D}{D}_{\mu} \phi\right)\left(\bar{d}_{R 3} \gamma^{\mu} d_{R 3}\right)$ & $\mathcal{O}_{\phi t}$ & $\left(\phi^{\dagger} i \stackrel{\leftrightarrow}{D}_{\mu} \phi\right)\left(\bar{u}_{R 3} \gamma^{\mu} u_{R 3}\right)$ \\
$\mathcal{O}_{\phi q}^{(1)}$ & $\left(\phi^{\dagger} i \stackrel{D}{D}_{\mu} \phi\right)\left(\bar{q}_{L 3} \gamma^{\mu} q_{L 3}\right)$ & $\mathcal{O}_{\phi q}^{(3)}$ & $\left(\phi^{\dagger} i \stackrel{D}{D}_{\mu}^{a} \phi\right)\left(\bar{q}_{L 3} \gamma^{\mu} \sigma_{a} q_{L 3}\right)$ \\
$\mathcal{O}_{\phi t b}$ & $\left(\tilde{\phi}^{\dagger} i D_{\mu} \phi\right)\left(\bar{u}_{R 3} \gamma^{\mu} d_{R 3}\right)$ & & \\
$\mathcal{O}_{b B}$ & $\left(\bar{q}_{L 3} \sigma^{\mu \nu} d_{R 3}\right) \phi B_{\mu \nu}$ & $\mathcal{O}_{t B}$ & $\left(\bar{q}_{L 3} \sigma^{\mu \nu} u_{R 3}\right) \tilde{\phi} B_{\mu \nu}$ \\
$\mathcal{O}_{b W}$ & $\left(\bar{q}_{L 3} \sigma^{\mu \nu} d_{R 3}\right) \sigma^{a} \phi W_{\mu \nu}^{a}$ & $\mathcal{O}_{t W}$ & $\left(\bar{q}_{L 3} \sigma^{\mu \nu} u_{R 3}\right) \sigma^{a} \tilde{\phi} W_{\mu \nu}^{a}$ \\
$\mathcal{O}_{b G}$ & $\frac{1}{2}\left(\bar{q}_{L 3} \sigma^{\mu \nu} \lambda^{A} d_{R 3}\right) \phi G_{\mu \nu}^{A}$ & $\mathcal{O}_{t G}$ & $\frac{1}{2}\left(\bar{q}_{L 3} \sigma^{\mu \nu} \lambda^{A} u_{R 3}\right) \tilde{\phi} G_{\mu \nu}^{A}$ \\
\hline
\end{tabular}

Table 5. Dimension-6 operators generated by tree-level matching of the effective theory with each multiplet to the SMEFT. This basis of operators was proposed in [38] and is a subset of the Warsaw basis [39].

be neglected for RVLQ. However, for NRVLQ, the dimension-5 contribution is the leading one. Following the method in ref. [19], we can use the experimental measurements of $R_{b}$, $A_{F B}^{b}, A_{b}$ and $R_{c}$ to obtain the following bounds: $s_{L}<0.13$ for the triplet $T_{4}, s_{L}^{d}<0.02$ for the quadruplet $F_{7}$ and $s_{L}^{d}<0.03$ for the quadruplet $F_{5}$. These limits are already satisfied 
by the mixing angles

$$
\theta \sim \frac{y v^{2}}{M} \lesssim 0.02
$$

for $y \leq(3 \mathrm{TeV})^{-1}, M \geq 1 \mathrm{TeV}$. The quadruplet $F_{1}$ produces a $Z b b$ coupling with an extra suppression of $m_{b} / M$, so it is even less constrained. The limits from $S$ and $T$ are weaker than the ones from $Z \rightarrow b b$ when there is a $B$ component in the multiplet. The only multiplet without such component among the non-renormalizable ones is $T_{5} \sim 3_{5 / 3}$. In this case both the limits from $Z \rightarrow b b$ and from $S$ and $T$ may be relevant. Anyway, since these effects are loop suppressed, as long as $y / M \leq(1.7 \mathrm{TeV})^{-2}$, this multiplet satisfies these constraints.

Higgs physics. The $\mathcal{O}_{t \phi}$ operator introduces a modification of the top Yukawa coupling, which can be measured using $t t H$ production. This process has been observed at the LHC [40, 41]. The current uncertainty for the top Yukawa coupling is however too large for the effects of $\mathcal{O}_{t \phi}$ to be relevant. The situation could improve in future experiments [42].

The presence of $\mathcal{O}_{t \phi}$ also changes gluon fusion Higgs production, through its appearance in diagrams with loops of the top quark. In addition, there are contributions to $g g \rightarrow$ $H$ from the heavy-quark loops. At the renormalizable level, the contribution of the $T$ loops is cancelled quite precisely by the effect of $t$ loops with insertions of $\mathcal{O}_{t \phi}$ (such cancellation does not happen for $B$ loops) [19]. In the presence of $Q q \phi \phi$ operators the cancellation is spoiled by the contributions to $\mathcal{O}_{t \phi}$ proportional to $\lambda y$. However, this contribution is suppressed not only by $M / \Lambda$ but also by the small mixing. The dimension5 interactions with $Y_{1}$ give yet another contribution to this process (see also ref. [29]). This can be computed by one-loop matching to the SMEFT. The relevant part of the effective Lagrangian is

$$
\mathcal{L}_{1 \text {-loop }} \supset \frac{(2 T+1) \alpha_{s} \operatorname{Re}\left(Y_{1}\right)}{12 \pi M} \mathcal{O}_{\phi G},
$$

where $\mathcal{O}_{\phi G}=\phi^{\dagger} \phi G_{\mu \nu}^{A} G^{A, \mu \nu}$. As we can see, the coefficient of the induced operator is not suppressed by the mixing. Bounds on the coefficient on this operator have been calculated in ref. [43]. They can be translated into limits for the parameters of our theory:

$$
\frac{\left|\operatorname{Re}\left(Y_{1}\right)\right|}{M}<\frac{1}{(2 T+1)(1.25 \mathrm{TeV})^{2}}
$$

where $T$ is the isospin of the corresponding multiplet. Of course, both $\mathcal{O}_{\phi G}$ and $\mathcal{O}_{t \phi}$ contribute to the $H \rightarrow g g$ partial width, through tree-level and one-loop diagrams, respectively. This is discussed in detail in ref. [19]. These operators modify also double Higgs production, which has not been observed yet but could be measured at the HL-LHC [44]. Similarly, there are loop contributions to other vector-boson decay modes of the Higgs.

On the other hand, the $H \rightarrow b b$ decay channel is modified at the tree level by the operator $\mathcal{O}_{b \phi}$. Because the contribution to this operator from dimension-4 couplings is suppressed by the Yukawa coupling of the bottom quark, while the dimension- 5 contribution does not contain this suppression, it is possible that the dimension-5 interaction dominates. Using the limit on the coefficient of $\mathcal{O}_{b \phi}$ from ref. [43] (with milder flavour assumptions), we find the bound $\left|y_{(b)}\right| / M \lesssim(0.2 \mathrm{TeV})^{-2}$. 


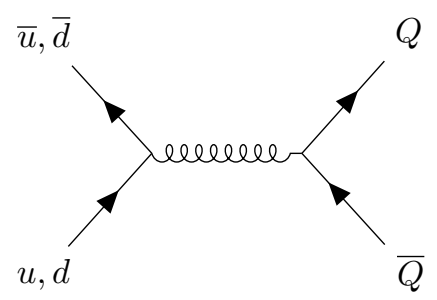

(a)

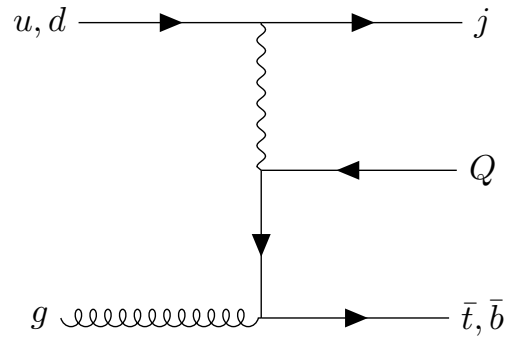

(b)

Figure 3. Production of heavy quarks in hadron colliders: (a) example diagram for pair production; (b) single production in association with a light jet $j$ and a heavy Standard Model quark $q=t, b$.

Top physics. Several of the dimension-6 SMEFT operators generated at tree level are relevant for the production of the top quark. $\mathcal{O}_{t W}$ and $\mathcal{O}_{\phi q}^{(3)}$ contribute to single production, whereas $\mathcal{O}_{t G}$ contributes to pair production [45]. In ref. [46], upper limits on the coefficients of these operators are derived. They range from approximately $(0.5 \mathrm{TeV})^{-2}$ to $(0.8 \mathrm{TeV})^{-2}$. Again, the natural values of these coefficients in our case, which are given by $\sim \lambda^{2} / 2 M$ and $\sim \lambda w / M$, already satisfy these limits. The same happens for the operators $\mathcal{O}_{t B}, \mathcal{O}_{\phi t}$ and $\mathcal{O}_{\phi q}^{(1)}$, which contribute to $t t \gamma$ and $t t Z$ production, and have even weaker limits.

Low-energy CP violation. The imaginary part of the coefficients of the operators $\mathcal{O}_{t \phi}$, $\mathcal{O}_{\phi t b}, \mathcal{O}_{t W}, \mathcal{O}_{t B}, \mathcal{O}_{b W}$ and $\mathcal{O}_{t G}$ affects the electric dipole moment of the electron and the neutron. These low-energy observables must be computed by performing the RG running of the coefficients down to the electroweak scale and integrating out the top quark. In refs. [47-49], strong limits on the imaginary part of the coefficients have been obtained, ranging from $(2 \mathrm{TeV})^{-2}$ to $(42 \mathrm{TeV})^{-2}$. Our UV parameters enter these coefficients with the combination $\lambda w^{*} / M$, so either their absolute value is very small, or all their phases must be almost equal. A trivial way of satisfying these limits is by imposing that all parameters are real.

\section{Production at the $\mathrm{LHC}$}

All the vector-like quarks can be produced in pairs at hadron colliders by their coupling to gluons, which is determined by the value of $\alpha_{s}$ at the relevant energy. Given $M$, the production cross section is fixed and it is the same for all the multiplets. One of the several tree-level diagrams contributing to pair production is represented in figure $3(\mathrm{a})$. On the other hand, the $T, B$ states can be singly produced via their mixing with the SM $t, b$ quarks. The corresponding process is represented in figure 3(b).

When the heavy quarks have low mass, the cross section for pair production is larger than the one for single production. As their mass increases, and for fixed collider energy, the later eventually becomes the main production mechanism. This has been studied for RVLQ mixing with the third family in ref. [19]. For these multiplets, the addition of dimension-5 interactions with natural values of the $y$ couplings and $\Lambda \geq 2 \mathrm{TeV}$ does not 

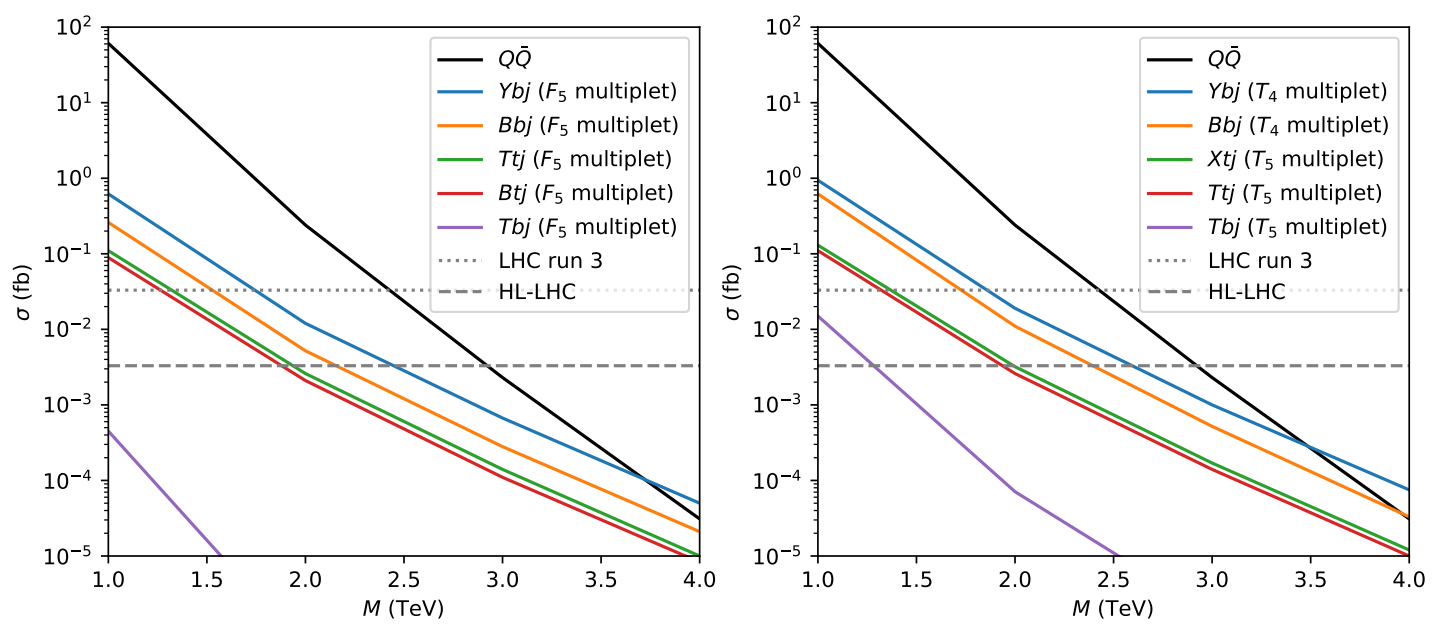

Figure 4. Cross section for different processes for production of heavy quarks with $y=(4 \mathrm{TeV})^{-1}$ and a center-of-mass energy of $14 \mathrm{TeV}$. The left plot corresponds to the $F_{5}$ quadruplet, while the right plot is for the $T_{5}$ and $T_{4}$ triplets. Pair production dominates for masses below $\simeq 3.5 \mathrm{TeV}$. The dotted and dashed gray lines represent the minimum cross section needed to obtain at least 10 events at the corresponding collider, assuming that the expected integrated luminosity is reached [50].

change significantly the results, as they give a small correction to the cross section. Here we are assuming that the dimension- 4 couplings saturate the electroweak limits. In the case of NRVLQ, for natural values of the $y$ couplings and $\Lambda \geq 2 \mathrm{TeV}$, pair production is larger than single production for the range of masses that can be tested at colliders in the present and near future. Some examples of the dependence of the production cross section on the the mass are shown in figure 4 .

The operators $\bar{Q} \sigma^{\mu \nu} q F_{\mu \nu}$ open new single production channels, which are suppressed by $(M / \Lambda)^{2}$ instead of $\sin ^{2} \theta$. In figure 5 , we show the two main mechanisms, which produce a heavy quark in association with a Standard Model third generation quark. Other single production processes are possible with $b$ quarks from the protons in the initial state. In this way, the $B$ component of multiplets with these operators can be generated alone, while the $T$ component can be produced together with a jet or a $W$ boson. As an example, we show in figure 6 the cross section of the $T$ production processes involving these operators, for the $U$ multiplet. For $w=(4 \mathrm{TeV})^{-1}$ these cross sections are large. However, these couplings are generated in renormalizable UV completions only at one loop, so the natural value for $w$ is expected to have a suppression of $1 / 16 \pi^{2}$ in weakly coupled UV completions. Including this suppression gives cross sections that are smaller than pair production.

A concrete model with $\bar{Q} \sigma^{\mu \nu} q F_{\mu \nu}$ operators has been tested experimentally, as presented in ref. [51], for the case of the multiplet $Q_{1}$. This analysis focuses on a particular direction in parameter space, which in our notation corresponds to: $g_{s} w^{G}=g w^{W}=$ $-g^{\prime} w^{B} / 6$, with the coefficients of all the other operators set to zero. The search is for the decay into $\gamma b$. Under these conditions, $M$-dependent limits over the coefficients of the operators have been obtained, for masses between $M=1 \mathrm{TeV}$ and $M=1.8 \mathrm{TeV}$. Translated into our notation, the bounds for these two masses are $w^{G} \lesssim(7 \mathrm{TeV})^{-1}$ and $w^{G} \lesssim(5 \mathrm{TeV})^{-1}$, respectively. 

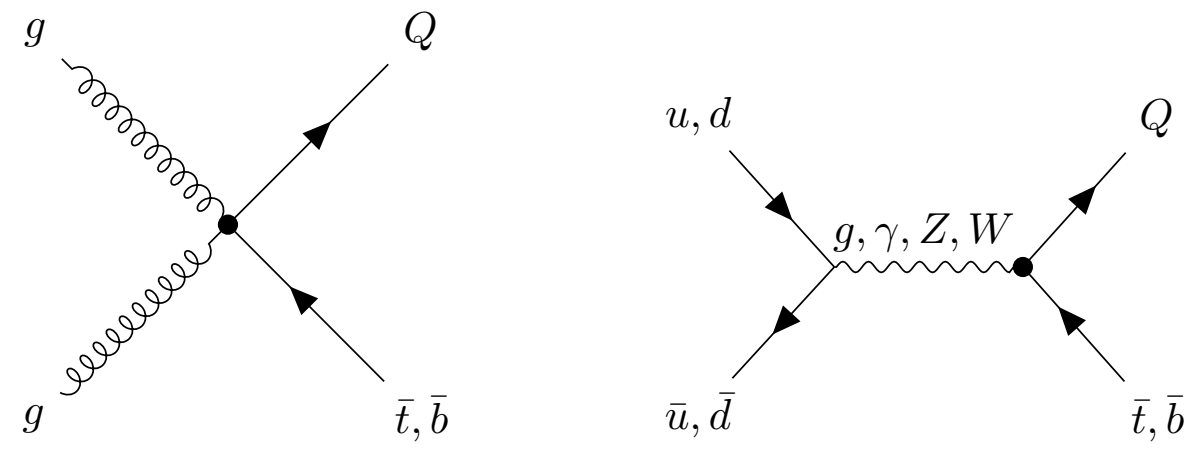

Figure 5. Single production with $\bar{Q} \sigma^{\mu \nu} q F_{\mu \nu}$-type operators.

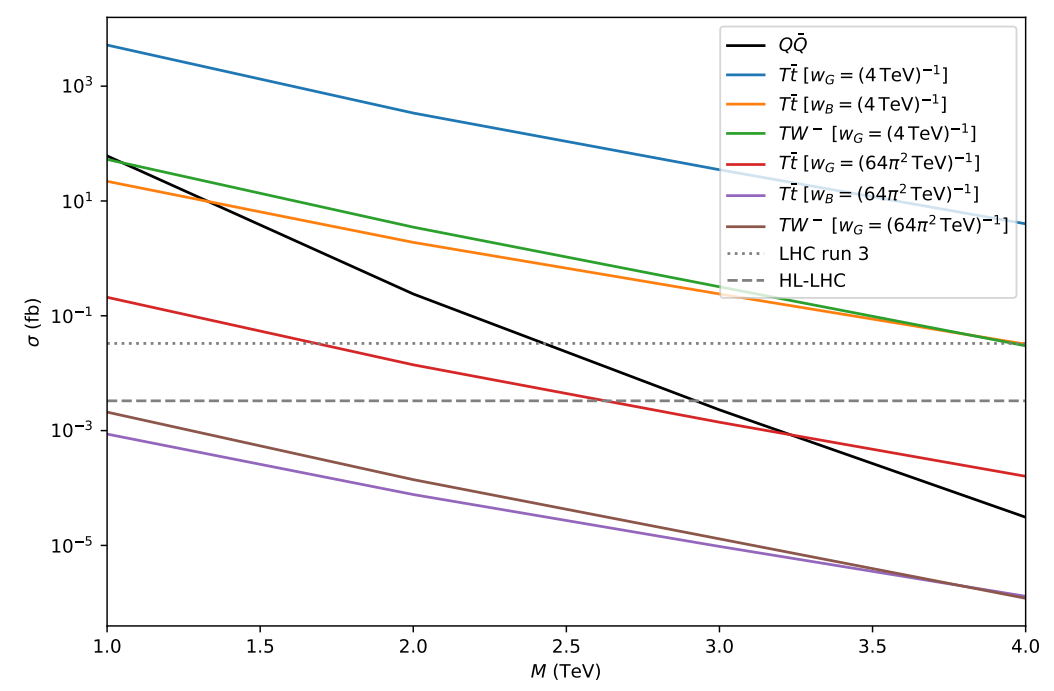

Figure 6. Cross section for different processes involving $\bar{Q} \sigma^{\mu \nu} q F_{\mu \nu}$, for production of heavy quarks in the $U$ model, with a center-of-mass energy of $14 \mathrm{TeV}$.

\section{Decay}

In this section, we study the decays of the heavy quarks. Barring cancellations with other heavy physics, electroweak precision tests require small mixings. In this case, the splittings between the different components of the extra quark multiplet are small (of a few $\mathrm{GeV}$ at most for masses below $2 \mathrm{TeV}$ ). This in turn implies that the decays from one component to another are very suppressed. The $T$ and $B$ states can decay via mixing into $H t, Z t, W^{+} b$ and $H b, Z b, W^{-} b$, respectively. They can also decay into $t \gamma, t g$ and $b \gamma, b g$, respectively, in the presence of $w$ couplings. The $X$ and $Y$ states decay via mixing mainly into $W^{+} t$ and $W^{-} b$, respectively. Their three-body decays are also sizable. Finally, $X^{\prime}$ and $Y^{\prime}$ have no two-body decays, as their charges differ by at least two units from the ones of the SM quarks.

The decay width of RVLQ is typically large enough for them to have prompt decays and small enough for a good narrow width approximation. The NRVLQ, on the other hand, have smaller and smaller widths for larger and larger values of the cutoff $\Lambda$. In figure 7 , we 
show the dependence of the total width of $T$ and $B$ with the dimension-5 Yukawa coupling $y$ for each type of NRVLQ, for $M=2 \mathrm{TeV}$. For widths below the QCD scale (see the discussion below), we have extrapolated the results calculated for larger couplings.

For small enough widths, i.e. long lifetimes, the phenomenology of the vector-like quarks can be completely different from the one in the standard searches of these particles. First, when the width is smaller than the QCD scale $\Lambda_{\mathrm{QCD}}$, non-perturbative effects, including hadronization, will be significant before the quarks have time to decay. One possibility is the formation near threshold of $Q \bar{Q}$ quarkonium states. This has been studied in ref. [52] (see also the review in ref. [53]) and generalized in ref. [34] to higher color representations. Possible signatures would have di-photon and di-lepton resonant final states. But the production cross-section is suppressed by the wave function at the origin and the cross sections are small. For instance, for $M$ above at the $0.01 \mathrm{TeV}$, ref. [34] shows that the cross section into $\gamma \gamma$ for quarks with masses above $1 \mathrm{TeV}$ is below $0.01 \mathrm{fb}$. In fact, most of the time the heavy quarks will fragment independently forming $Q q$ meson states and also baryons with light quarks from the vacuum. This is completely analogous to the case of $b$-quarks forming $B$ mesons. For $M \gg \Lambda_{\mathrm{QCD}}$, the mass and partial decay widths of the hadrons will inherit the properties of the heavy quark, up to small QCD corrections. Moreover, most of the energy resides in the hadron containing the heavy quark, leaving only a small fraction to light particles in the accompanying jet, and gluon radiation only softens the spectrum slightly [52]. Hence, the standard type of search for vectorlike quarks will be mostly blind to the fact that the quarks hadronize, as long as they decay promptly (that is, for lifetimes below $10^{-14} \mathrm{~s}$ ).

For widths smaller than $\sim 10^{-12} \mathrm{GeV}$, the hadrons carrying the heavy quark will be long-lived. In this context, they are called $R$-hadrons. Their phenomenology at the LHC has been studied in detail, especially for squarks and gluinos in supersymmetric models. $R$-hadrons interact hadronically as they move through the detector, but in these processes the heavy quark acts mostly as a spectator of the low-energy scattering of light partons. Compared to SM hadrons, their energy loss in the calorimeter is small. Possible signatures include (see ref. [54] for a review of the phenomenology of long-lived particles):

- Tracks with anomalous ionization, from the slower speed of the heavy quarks in comparison to SM particles and/or non-standard charges. Note that $Q q$ mesons formed with $X, X^{\prime}, Y$ or $Y^{\prime}$ will always be charged, while those with $T$ and $B$ can be charged or neutral. In these searches, one must take into account the fact that the charge of the $R$-hadrons may change due to the hadronic interactions of the light partons with the detector material.

- Delayed detector signals, due again to the small speed. In the extreme case, it is possible for a quasi-stable $R$-hadron to loose all its energy and stop at the hadronic calorimeter; its eventual decay would give out-of-time signals.

- Displaced vertices from the delayed decay of the heavy quark. The final states produced by $R$-hadrons with vector-like quarks are very different from the ones in super- 


\begin{tabular}{|cccccccc|}
\hline \multicolumn{9}{c|}{$\Lambda_{\mathrm{QCD}}$} & \multicolumn{3}{c|}{$\Lambda_{\text {disp }}$} & $\Lambda_{\text {long lived }}$ \\
& $T_{4}$ & $T_{5}$ & $F_{1}$ & $F_{5}$ & $F_{7}$ & & \\
\hline$X^{\prime}$ & - & 1.0 & - & - & 1.0 & $5 \times 10^{5}$ & $5 \times 10^{7}$ \\
$X$ & - & 4.6 & 3.1 & - & 3.9 & $10^{6}$ & $10^{8}$ \\
$T$ & - & 5.3 & 3.7 & 5.7 & 5.6 & $10^{6}$ & $10^{8}$ \\
$B$ & 5.3 & - & 3.7 & 5.7 & 5.7 & $10^{6}$ & $10^{8}$ \\
$Y$ & 4.6 & - & 3.1 & 3.9 & - & $10^{6}$ & $10^{8}$ \\
$Y^{\prime}$ & 1.1 & - & - & 1.1 & - & $5 \times 10^{5}$ & $5 \times 10^{7}$ \\
\hline
\end{tabular}

Table 6. Value of $1 / y$ (in TeV) at which the total width reaches the scales $\Lambda_{\mathrm{QCD}}=0.2 \mathrm{GeV}$, $\Lambda_{\text {disp }}=10^{-12} \mathrm{GeV}$ and $\Lambda_{\text {long lived }}=10^{-16} \mathrm{GeV}$. For $\Lambda_{\text {disp }}$ and $\Lambda_{\text {long lived }}$ only an estimate of the order of magnitude is provided, obtained by extrapolation of the results above $\Lambda_{\mathrm{QCD}}$.

symmetric theories and other scenarios considered thus far. So, a dedicated search for displaced vertices of vector-like quarks would be necessary to probe this scenario.

The relevance of each of the signatures depends crucially on the lifetime of the $R$-hadron, which is of the order of the lifetime of the heavy quark, as calculated ignoring QCD. In table 6 , we give the values of $1 / y$ above which i) non-perturbative QCD is important $\left(\Lambda_{\mathrm{QCD}}\right)$, ii) displaced vertices can be observed $\left(\Lambda_{\text {disp }}\right)$ and iii) the heavy quark is stable within detector distances $\left(\Lambda_{\text {long lived }}\right)$.

As a reference, ATLAS has recently put bounds on the mass of long-lived supersymmetric $R$-hadrons, using ionization energy loss and time-of-flight information [55]. This search is quite model-independent and can be adapted to the case of vector-like quarks (which are also color-triplets but fermions, rather than scalars). Comparing with the limits on production cross sections for squarks and sgluinos, we estimate a lower bound close to $1500 \mathrm{GeV}$ on the mass of detector stable vector-like quarks.

In the following we concentrate on branching ratios, having in mind mostly the case with prompt decays. Consider RVLQ. If the dimension-5 couplings are turned off, $T$ essentially decays only into $H t, Z t$ or $W^{+} b$, while $B$ decays into $H b, Z t$ or $W^{-} t$. Changing the specific values of the parameters in these models has a small effect in the branching ratios. This means that the branching ratios are approximately determined by the choice of multiplet. Because the sum of branching ratios must be one

$$
B R(Q \rightarrow H q)+B R(Q \rightarrow Z q)+B R\left(Q \rightarrow W^{ \pm} q^{\prime}\right)=1,
$$

(with $Q=T, B$ and $q, q^{\prime}=t, b$ ) it suffices to know two branching ratios of $Q$ to be able to know the third. Any two branching ratios $B R_{1}$ and $B R_{2}$ of $Q$ form a point in the triangle $B R_{1}+B R_{2} \leq 1, B R_{1,2} \geq 0$. Thus, each multiplet determines a point in this triangle (or a short segment, taking into account variations of the values of the parameters). This is the usual method for representing graphically the branching ratios of vector-like quarks [56].

The addition of dimension- 5 interactions modifies these points, both by changing the corresponding partial widths and by introducing new decay channels. Then, eq. (6.1) no longer holds. For any choice of the values of the parameters, the branching ratios define 
a point $p$ in the multi-dimensional simplex determined by $B R_{i} \leq 1, \sum_{i} B R_{i}=1$. In particular, the branching ratios into $H t, Z t$ and $W^{+} b$ define a point that falls inside the tetrahedron

$$
\begin{array}{r}
\Sigma:=B R(Q \rightarrow H q)+B R(Q \rightarrow Z q)+B R\left(Q \rightarrow W^{ \pm} q^{\prime}\right) \leq 1, \\
B R(Q \rightarrow H q), B R(Q \rightarrow Z q), B R\left(Q \rightarrow W^{ \pm} q^{\prime}\right) \geq 0 .
\end{array}
$$

For their graphical representation, we have chosen to plot the projections of $p$ into the $B R(Q \rightarrow Z q)-B R(Q \rightarrow H q)$ plane and into the $B R\left(Q \rightarrow W^{ \pm} q^{\prime}\right)-B R(Q \rightarrow H q)$ plane, as shown in figure 8 .

The results for RVLQ are presented in figures 10,11, 12 and 13, while the branching ratios of NRVLQ are presented in figures 14, 15, 16 and 17. Each segment is obtained by evaluating at $M=1 \mathrm{TeV}$ and at $M=2 \mathrm{TeV}$ while keeping all the other parameters fixed. The value of the coefficients of dimension- 5 operators is chosen to be $(2 \mathrm{TeV})^{-1}$. This pretty large value has been chosen to visually highlight the directions of the corrections induced on the branching ratios for RVLQ. For lower, probably more realistic values of the coefficients (especially for $w$ ), these corrections will be smaller. For the multiplets without dimension-4 interactions, this value of the coefficients ensures that the decay width is much higher than the QCD scale, so that that QCD effects can be neglected. The branching ratios do not change much with the value of the corresponding coefficient in the range from $(2 \mathrm{TeV})^{-1}$ down to the values in which the total width equals $\Lambda_{\mathrm{QCD}}$. As it can be clearly seen in the figures, most branching ratios points lie near or directly over the $B R(Q \rightarrow H q)=B R(Q \rightarrow Z q)$ diagonal. This happens in all cases where the coefficients of the $\bar{Q} \sigma^{\mu \nu} q F_{\mu \nu}$-type operators vanish, except for the $F_{1}$ multiplet. An explanation for this fact is given in appendix C.

The experimental analyses of searches of pair-produced vector-like quarks usually combine the information on the different final states to put lower bounds on the heavy quark masses, as a function of the branching ratios to $W q^{\prime}, Z q$ and $H q$ [57, 58]. Eq. (6.1) is assumed in these analyses, so the results are not directly valid beyond the renormalizable level. However, they can be adapted to the case where other decay channels are present. This has been discussed previously in refs. $[33,59]$. In appendix $\mathrm{B}$ we derive a simple formula for the corrected mass limit due to the presence of extra decays:

$$
M_{\Sigma}=\left(M_{1}^{1 / 2}+f^{1 / 2} \log \Sigma\right)^{2}
$$

It gives a lower bound $M_{\Sigma}$ on the mass of any heavy quark as a function of the lower bound $M_{1}$ it would have if its branching ratios into $H t, Z q$ and $W^{ \pm} q^{\prime}$ were rescaled by the same factor $1 / \Sigma$, such that eq. (6.1) holds. Here, $f=20.5 \mathrm{GeV}$ is just a constant. In table 7 , we present the limits calculated using this formula, for different choices of the values of the parameters for each model, taking the bound $M_{1}$ from ref. [57]. In all cases the couplings of dimension-4 operators are chosen to saturate the electroweak limits. In figure 9, we show the corrections induced by the use of this formula on the results of ref. [57], for the value $\Sigma=1 / 2$. 


\begin{tabular}{|cc|}
\hline \multicolumn{2}{|c|}{$U \sim 1_{2 / 3}$} \\
\hline Only $\operatorname{dim} .4$ & 1300 \\
$y$ & 1310 \\
$w_{B}$ & 1010 \\
$w_{G}$ & $<800$ \\
\hline
\end{tabular}

\begin{tabular}{|cc|}
\hline \multicolumn{2}{|c|}{$D \sim 1_{2 / 3}$} \\
\hline Only dim. 4 & 1200 \\
$y$ & 1190 \\
$w_{B}$ & $<800$ \\
$w_{G}$ & $<800$ \\
\hline
\end{tabular}

\begin{tabular}{|cc|}
\hline \multicolumn{2}{|c|}{$Q_{1} \sim 2_{1 / 6}$} \\
\hline Only dim. 4 & 1340 \\
$y_{t}$ & 1340 \\
$y_{b}$ & 1120 \\
$w_{B}$ & 830 \\
$w_{W}$ & 1250 \\
$w_{G}$ & $<800$ \\
\hline
\end{tabular}

\begin{tabular}{|cr|}
\hline \multicolumn{2}{|c|}{$Q_{5} \sim 2_{-5 / 6}$} \\
\hline Only dim. 4 & 1130 \\
$y$ & 1130 \\
\hline
\end{tabular}

\begin{tabular}{|cc|}
\hline \multicolumn{2}{|c|}{$Q_{7} \sim 2_{7 / 6}$} \\
\hline Only dim. 4 & 1360 \\
$y$ & 1350 \\
\hline
\end{tabular}

\begin{tabular}{|cc|}
\hline \multicolumn{2}{|c|}{$T_{1} \sim 3_{-1 / 3}$} \\
\hline Only dim. 4 & 1220 \\
$y_{t}$ & 1250 \\
$y_{b}$ & 1200 \\
$w$ & 970 \\
\hline
\end{tabular}

\begin{tabular}{|cc|}
\hline \multicolumn{2}{|c|}{$T_{2} \sim 3_{2 / 3}$} \\
\hline Only dim. 4 & 1130 \\
$y_{t}$ & 1130 \\
$y_{b}$ & 1130 \\
$w$ & 1260 \\
\hline
\end{tabular}

\begin{tabular}{|cr|}
\hline$T_{4} \sim 3_{-4 / 3}$ \\
\hline$y$ & 1130 \\
\hline
\end{tabular}

\begin{tabular}{|cr|}
\hline$T_{5} \sim 3_{5 / 3}$ \\
\hline$y$ & 1360 \\
\hline
\end{tabular}

\begin{tabular}{|cr|}
\hline \multicolumn{2}{|c|}{$F_{1} \sim 4_{1 / 6}$} \\
\hline$y$ & 1030 \\
$w$ & 1010 \\
\hline
\end{tabular}

\begin{tabular}{|cr|}
\hline$F_{5} \sim 4_{-5 / 6}$ \\
\hline$y$ & 1200 \\
\hline
\end{tabular}

\begin{tabular}{|cr|}
\hline$F_{7} \sim 4_{7 / 6}$ \\
\hline$y$ & 1130 \\
\hline
\end{tabular}

Table 7. Mass limits for each multiplet and different values of the couplings. In the right column, a lower bound on the mass of the heavy quark (in $\mathrm{TeV}$ ) is displayed, assuming that the corresponding coupling in the left column has a value of $(2 \mathrm{TeV})^{-1}$ and the other dimensionful couplings vanish. The dimensionless couplings $\lambda$ are always chosen to saturate the corresponding electroweak precision bounds.

We have emphasized the presence of alternative decay channels at the nonrenormalizable level. In tables 8 and 9 , we give the decay channels with branching ratio $>0.01$ other than $Z q, W^{ \pm} q^{\prime}$ and $H q$ for $T$ and $B$, together with the maximum value they get and the interaction that generates them. We choose the values $M=2 \mathrm{TeV}$ and $w, y=(2 \mathrm{TeV})^{-1}$, again quite extreme, in order to maximize these alternative branching ratios (including those of three-body decays). The tables also include three-body channels that survive when $w=y=0$. For RVLQ, the values of the couplings $\lambda$ are chosen to approximately saturate the electroweak limits. For smaller values of $\lambda$, the alternative channels will have larger branching ratios. Large branching ratios are found for channels involving "magnetic" operators. The reason is that the partial widths are suppressed in this case by $(M / \Lambda)^{2}$, in comparison with the $(v / M)^{2}$ suppression of the decay widths of 


\begin{tabular}{|c|c|c|c|}
\hline Multiplet & Decay products & Maximum $B R$ & Coupling \\
\hline \multirow{4}{*}{$U$} & $b \bar{b} t$ & 0.02 & $\lambda, y$ \\
\hline & $t t \bar{t}$ & 0.01 & $\lambda, y$ \\
\hline & $\gamma t$ & 0.71 & $w_{B}$ \\
\hline & $g t$ & 0.93 & $w_{G}$ \\
\hline \multirow{6}{*}{$Q_{1}$} & $t W^{+} W^{-}$ & 0.08 & $\lambda_{t}, y_{b}$ \\
\hline & $t t \bar{t}$ & 0.01 & $\lambda_{t}, y_{t}, y_{b}$ \\
\hline & $b H W^{+}$ & 0.11 & $y_{b}$ \\
\hline & $b Z W^{+}$ & 0.04 & $\lambda_{b}, y_{t}, y_{b}$ \\
\hline & $\gamma t$ & 0.77 & $w_{B}$ \\
\hline & $g t$ & 0.99 & $w_{G}$ \\
\hline$Q_{7}$ & $t W^{+} W^{-}$ & 0.08 & $\lambda$ \\
\hline \multirow{5}{*}{$T_{1}$} & $b H W^{+}$ & 0.10 & $y_{b}$ \\
\hline & $t W^{+} W^{-}$ & 0.07 & $\lambda, y_{t}, y_{b}$ \\
\hline & $b Z W^{+}$ & 0.83 & $w$ \\
\hline & $t t \bar{t}$ & 0.01 & $\lambda, y_{t}, y_{b}$ \\
\hline & $b \gamma W^{+}$ & 0.01 & $w$ \\
\hline \multirow{5}{*}{$T_{2}$} & $b H W^{+}$ & 0.17 & $y_{b}$ \\
\hline & $t W^{+} W^{-}$ & 0.25 & $w$ \\
\hline & $b Z W^{+}$ & 0.06 & $\lambda, y_{t}, y_{b}$ \\
\hline & $t b \bar{b}$ & 0.01 & $\lambda, y_{t}, y_{b}$ \\
\hline & $\gamma t$ & 0.21 & $w$ \\
\hline$T_{5}$ & $t W^{+} W^{-}$ & 0.08 & $y$ \\
\hline \multirow{4}{*}{$F_{1}$} & $b H W^{+}$ & 0.30 & $y$ \\
\hline & $b Z W^{+}$ & 0.23 & $w$ \\
\hline & $t W^{+} W^{-}$ & 0.66 & $w$ \\
\hline & $\gamma t$ & 0.09 & $w$ \\
\hline \multirow{2}{*}{$F_{5}$} & $b H W^{+}$ & 0.10 & $y$ \\
\hline & $t W^{+} W^{-}$ & 0.07 & $y$ \\
\hline \multirow{3}{*}{$F_{7}$} & $b H W^{+}$ & 0.28 & $y$ \\
\hline & $b Z W^{+}$ & 0.10 & $y$ \\
\hline & $t W^{+} W^{-}$ & 0.09 & $y$ \\
\hline
\end{tabular}

Table 8. Extra decay channels of $T$ with branching ratio larger than 0.01 for $M=2 \mathrm{TeV}$, when the couplings $\lambda$ are fixed to the values that saturate electroweak precision limits. The last column displays the coupling constant which, when set to $(2 \mathrm{TeV})^{-1}$, gives the maximum $B R$ in the corresponding channel. The appearance of $\lambda$ indicates that the channel in question is present already in the case with dimension-4 interactions only. 


\begin{tabular}{|c|c|c|c|}
\hline Multiplet & Decay products & Maximum $B R$ & Coupling \\
\hline \multirow{2}{*}{$D$} & $\gamma b$ & 0.77 & $w_{B}$ \\
\hline & $g b$ & 0.99 & $w_{G}$ \\
\hline \multirow{6}{*}{$Q_{1}$} & $t H W^{-}$ & 0.12 & $y_{t}$ \\
\hline & $t Z W^{-}$ & 0.04 & $\lambda_{t}, y_{t}, y_{b}$ \\
\hline & $b W^{+} W^{-}$ & 0.08 & $\lambda_{b}, y_{t}, y_{b}$ \\
\hline & $b t \bar{t}$ & 0.02 & $\lambda_{t}, y_{t}, y_{b}$ \\
\hline & $\gamma b$ & 0.77 & $w_{B}$ \\
\hline & $g b$ & 0.99 & $w_{G}$ \\
\hline \multirow{2}{*}{$Q_{5}$} & $b W^{+} W^{-}$ & 0.08 & $\lambda, y$ \\
\hline & $b t \bar{t}$ & 0.01 & $\lambda, y$ \\
\hline \multirow{5}{*}{$T_{1}$} & $t H W^{-}$ & 0.19 & $y_{t}$ \\
\hline & $t Z W^{-}$ & 0.06 & $\lambda, y_{t}, y_{b}$ \\
\hline & $b t \bar{t}$ & 0.01 & $\lambda, y_{t}, y_{b}$ \\
\hline & $b W^{+} W^{-}$ & 0.90 & $w$ \\
\hline & $\gamma b$ & 0.13 & $w$ \\
\hline \multirow{4}{*}{$T_{2}$} & $t H W^{-}$ & 0.10 & $\lambda$ \\
\hline & $b W^{+} W^{-}$ & 0.07 & $\lambda, y_{t}, y_{b}$ \\
\hline & $t Z W^{-}$ & 0.12 & $w$ \\
\hline & $b t \bar{t}$ & 0.02 & $\lambda, y_{t}, y_{b}$ \\
\hline$T_{4}$ & $b W^{+} W^{-}$ & 0.08 & $y$ \\
\hline \multirow{4}{*}{$F_{1}$} & $t H W^{-}$ & 0.30 & $y$ \\
\hline & $b W^{+} W^{-}$ & 0.69 & $w$ \\
\hline & $\gamma b$ & 0.09 & $w$ \\
\hline & $t Z W^{-}$ & 0.20 & $w$ \\
\hline \multirow{3}{*}{$F_{5}$} & $t H W^{-}$ & 0.28 & $y$ \\
\hline & $t Z W^{-}$ & 0.10 & $y$ \\
\hline & $b W^{+} W^{-}$ & 0.09 & $y$ \\
\hline \multirow{2}{*}{$F_{7}$} & $t H W^{-}$ & 0.10 & $y$ \\
\hline & $b W^{+} W^{-}$ & 0.07 & $y$ \\
\hline
\end{tabular}

Table 9. Extra decay channels of $B$ with branching ratio larger than 0.01 for $M=2 \mathrm{TeV}$, when the couplings $\lambda$ are fixed to the values that saturate electroweak precision limits. The last column displays the coupling constant which, when set to $(2 \mathrm{TeV})^{-1}$, gives the maximum $B R$ in the corresponding channel. The appearance of $\lambda$ indicates that the channel in question is present already in the case with dimension- 4 interactions only. 


\begin{tabular}{|cccc|}
\hline Multiplet & Decay products & Maximum $B R$ & Coupling \\
\hline \multirow{2}{*}{$Q_{7}$} & $t H W^{+}$ & 0.12 & $y$ \\
& $t Z W^{+}$ & 0.04 & $\lambda, y$ \\
\hline & $t H W^{+}$ & 0.10 & $\lambda, y_{d}$ \\
$T_{2}$ & $b W^{+} W^{+}$ & 0.04 & $\lambda, y_{u}, y_{d}$ \\
& $t Z W^{+}$ & 0.12 & $w$ \\
& $t t \bar{b}$ & 0.02 & $\lambda, y_{u}, y_{d}$ \\
\hline \multirow{2}{*}{$T_{5}$} & $t H W^{+}$ & 0.29 & $y$ \\
& $t Z W^{+}$ & 0.11 & $y$ \\
\hline \multirow{2}{*}{$F_{1}$} & $t H W^{+}$ & 0.32 & $y$ \\
& $t Z W^{+}$ & 0.82 & $w$ \\
\hline$F_{7}$ & $t H W^{+}$ & 0.32 & $y$ \\
\hline
\end{tabular}

Table 10. Decay channels of $X$ other than $W^{+} t$ with branching ratio larger than 0.01 for $M=$ $2 \mathrm{TeV}$, when the couplings $\lambda$ are fixed to the values that saturate electroweak precision limits. The last column displays the coupling constant which, when set to $(2 \mathrm{TeV})^{-1}$, gives the maximum $B R$ in the corresponding channel. The appearance of $\lambda$ indicates that the channel in question is present already in the case with dimension-4 interactions only.

\begin{tabular}{|cccc|}
\hline Multiplet & Decay products & Maximum $B R$ & Coupling \\
\hline \multirow{4}{*}{$Q_{5}$} & $b H W^{-}$ & 0.10 & $\lambda, y$ \\
& $b Z W^{-}$ & 0.04 & $\lambda, y$ \\
& $b b \bar{t}$ & 0.02 & $\lambda, y$ \\
\hline \multirow{2}{*}{$T_{1}$} & $b H W^{-}$ & 0.10 & $\lambda, y_{u}, y_{d}$ \\
& $b Z W^{-}$ & 0.83 & $w$ \\
& $t W^{-} W^{-}$ & 0.04 & $\lambda, y_{u}, y_{d}$ \\
\hline \multirow{2}{*}{$T_{4}$} & $b H W^{-}$ & 0.29 & $y$ \\
& $b Z W^{-}$ & 0.11 & $y$ \\
\hline \multirow{2}{*}{$F_{1}$} & $b H W^{-}$ & 0.32 & $y$ \\
& $b Z W^{-}$ & 0.82 & $w$ \\
\hline$F_{5}$ & $b H W^{-}$ & 0.32 & $y$ \\
\hline
\end{tabular}

Table 11. Decay channels of $Y$ other than $W^{-} b$ with branching ratio larger than 0.01 for $M=$ $2 \mathrm{TeV}$, when the couplings $\lambda$ are fixed to the values that saturate electroweak precision limits. The last column displays the coupling constant which, when set to $(2 \mathrm{TeV})^{-1}$, gives the maximum $B R$ in the corresponding channel. The appearance of $\lambda$ indicates that the channel in question is present already in the case with dimension- 4 interactions only.

standard channels. Note however that the value of $w$ we use is $\sim 16 \pi^{2}$ times too high in weakly coupled completions. A detailed analysis of the decays of a $U$ vector-like quark into $t \gamma$ and $t g$ at the LHC has been performed in ref. [60].

In the case of $X$ and $Y$, the decays into $W^{+} t$ and $W^{-} b$, respectively, have branching ratios in the range 60-90\%. The remaining decays are into three particles, two of which are always $W^{+} t$ or $W^{-} b$. The branching ratios for these channels are collected in tables 10 and 11. The states $X^{\prime}$ and $Y^{\prime}$ have only three-body decays. They always decay into $W^{+} W^{+} t$ and $W^{-} W^{-} b$, respectively. 

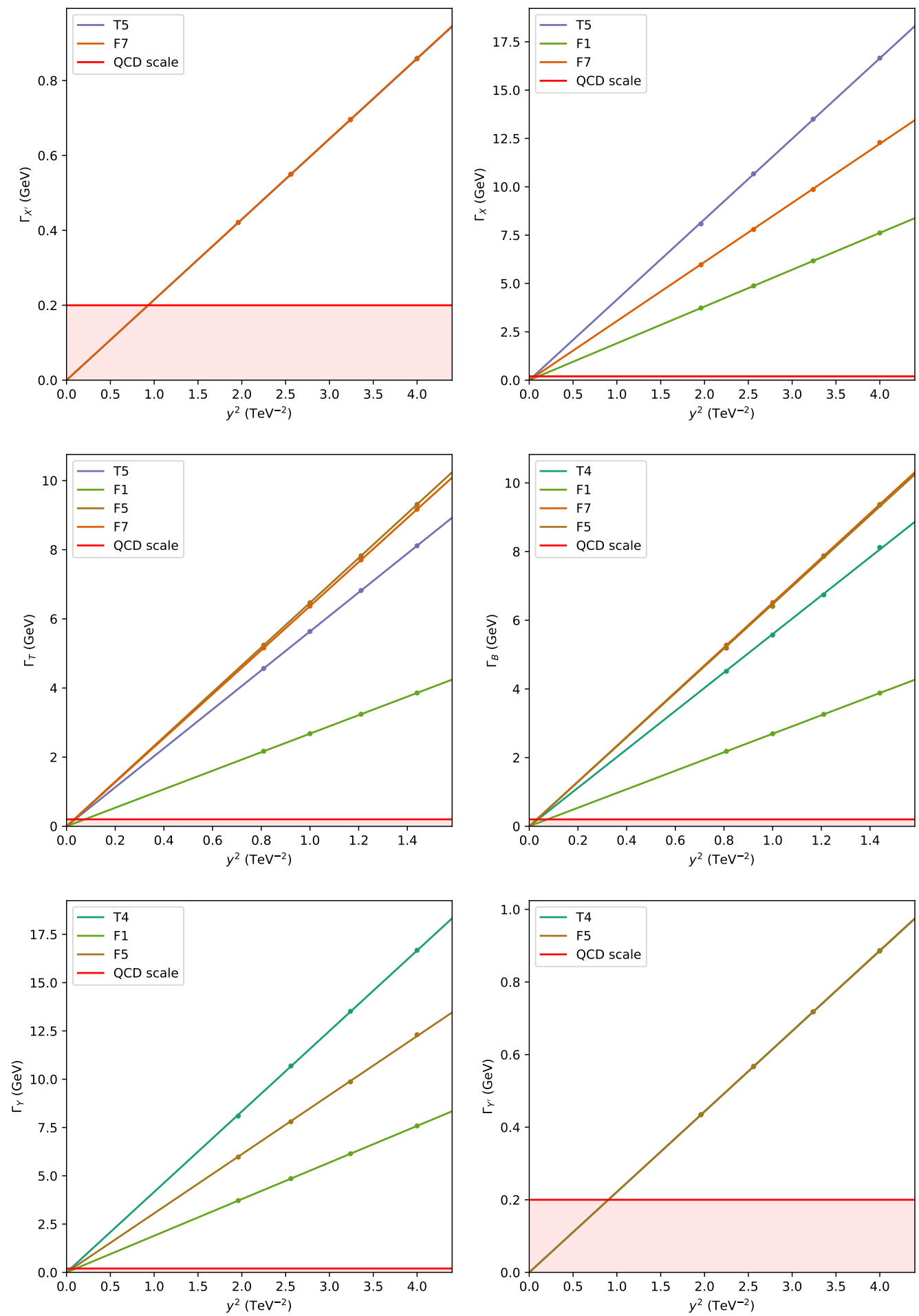

Figure 7. Total decay width of $T$ (left) and $B$ (right) vs the dimension-5 Yukawa coupling $y$ for each multiplet without dimension- 4 couplings and $M_{Q}=2 \mathrm{TeV}$. 


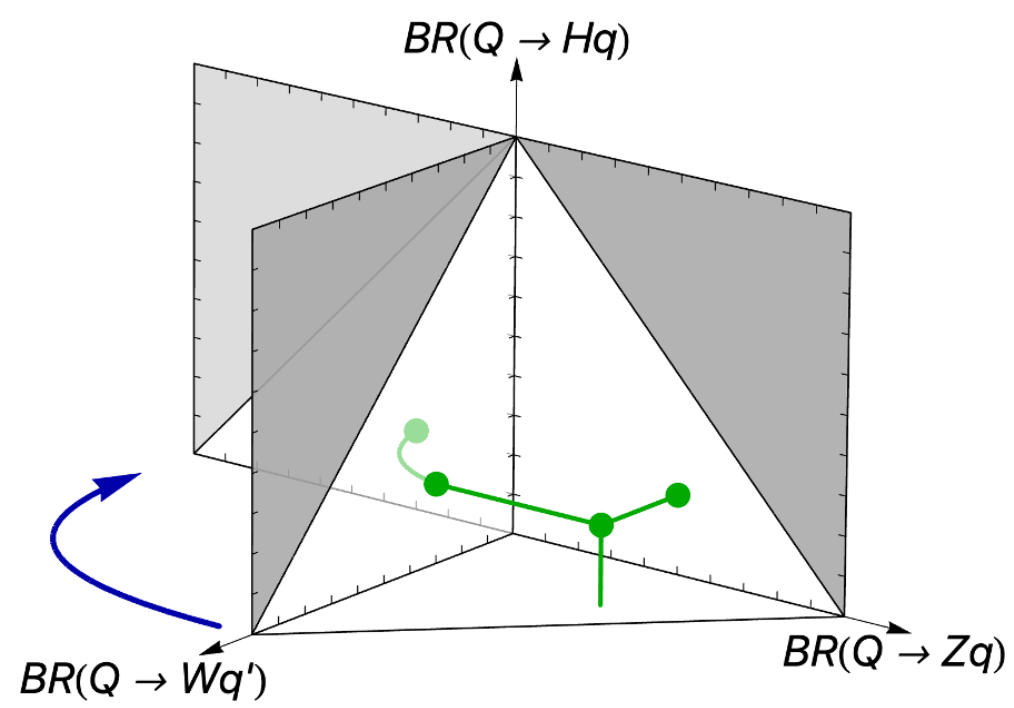

Figure 8. Representation of the $\left(B R(Q \rightarrow Z q), B R\left(Q \rightarrow W^{ \pm} q^{\prime}\right), B R(Q \rightarrow H q)\right)$ point as its projections into the $B R(Q \rightarrow Z q)-B R(Q \rightarrow H q)$ plane and into the $B R\left(Q \rightarrow W^{ \pm} q^{\prime}\right)-B R(Q \rightarrow H q)$ plane.
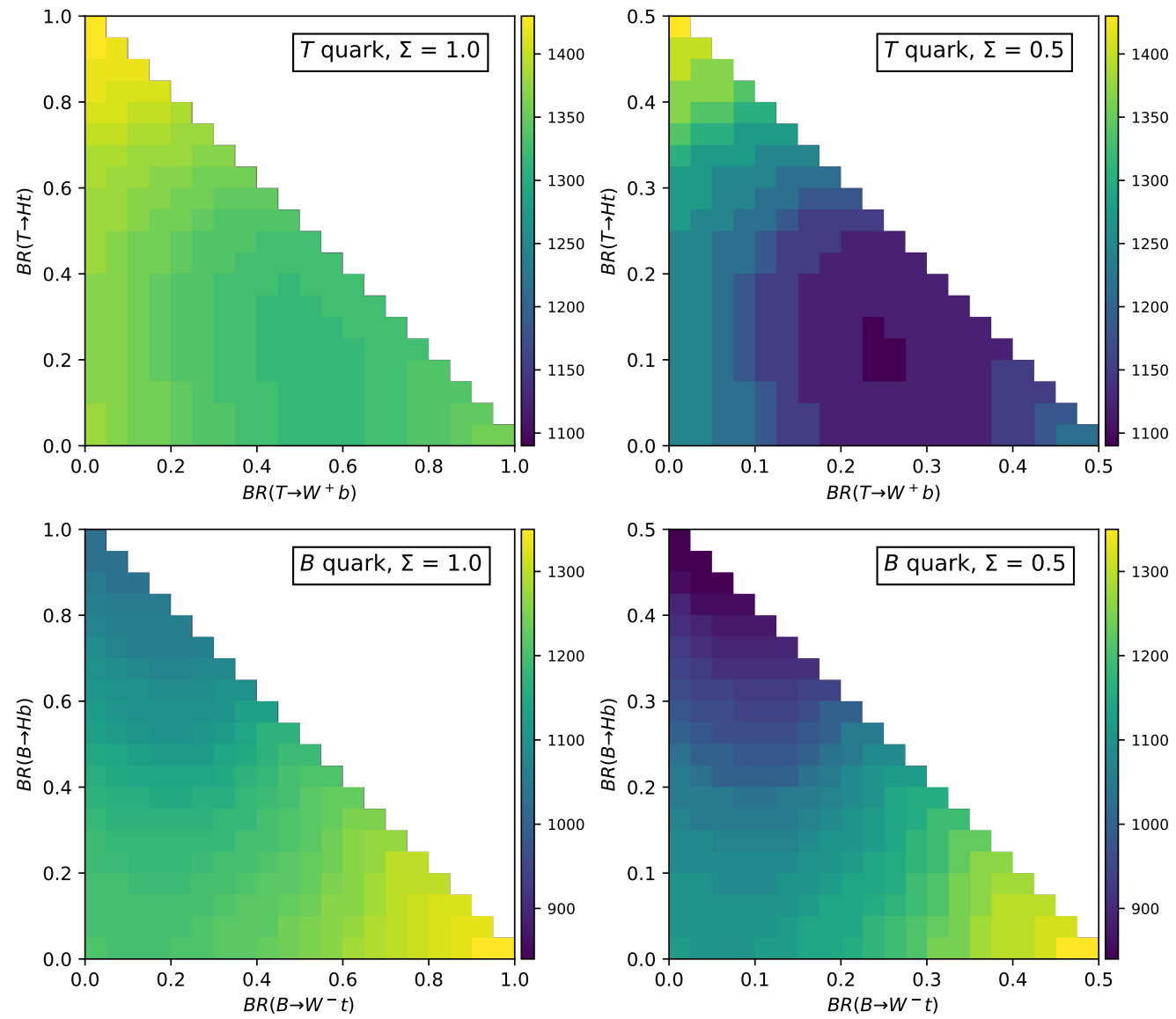

Figure 9. Left plots: lower bounds for the masses of heavy quarks presented in ref. [57] assuming that the sum of branching ratios into $H q, Z q$ and $W^{ \pm} q^{\prime}$ is $\Sigma=1$. Right plots: corrected lower bounds for the case in which $\Sigma=0.5$. 

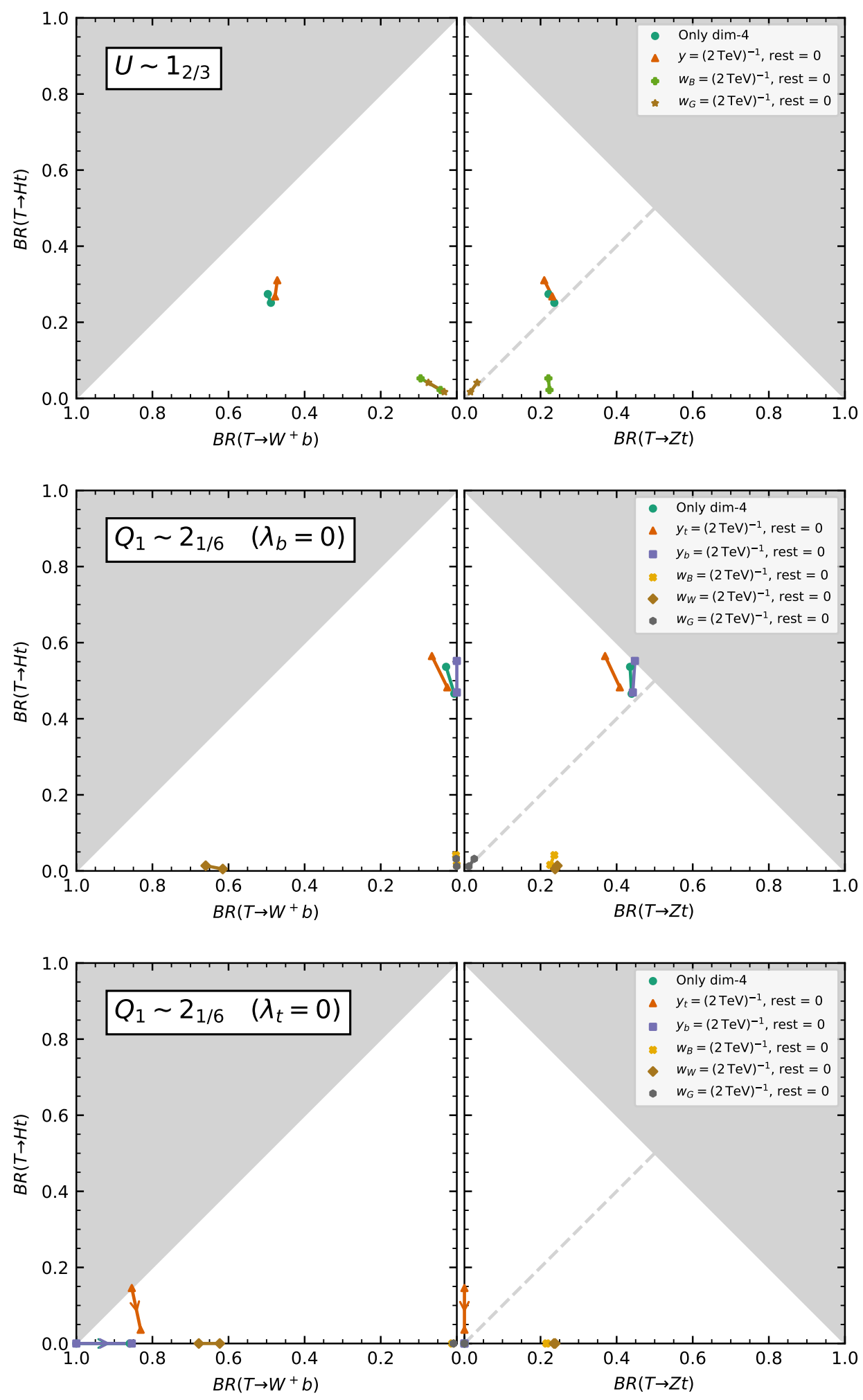

Figure 10. Branching ratios of $T$ into $H t, Z t$ and $W^{+} b$ for various values of the parameters in the $U, Q_{7}$ and $Q_{1}$ models. The dimensionless couplings $\lambda$ are always chosen to saturate the corresponding electroweak precision bounds. 

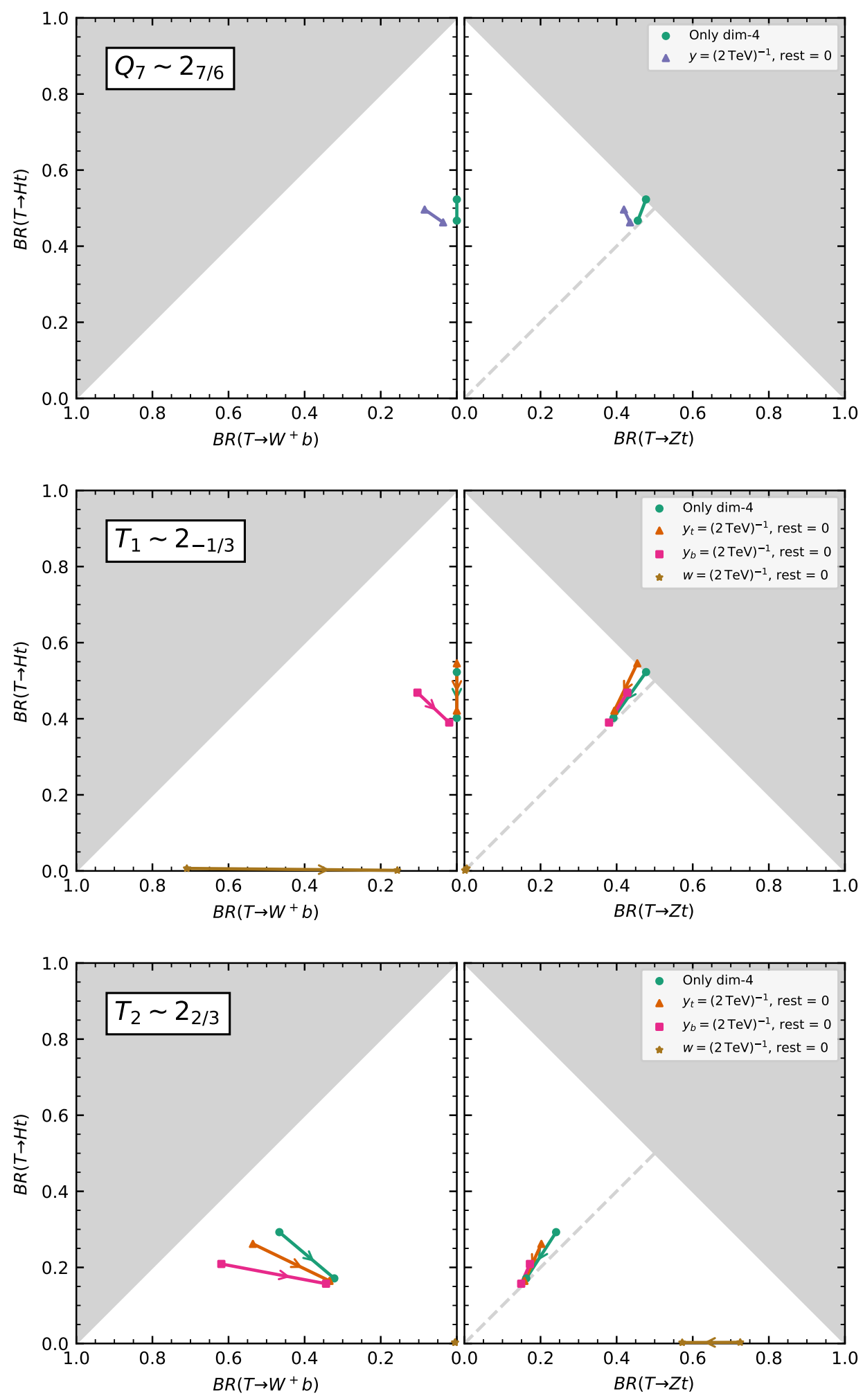

Figure 11. Branching ratios of $T$ into $H t, Z t$ and $W^{+} b$ for various values of the parameters in the $Q_{1}, T_{2}$ and $T_{1}$ models. The dimensionless couplings $\lambda$ are always chosen to saturate the corresponding electroweak precision bounds. 

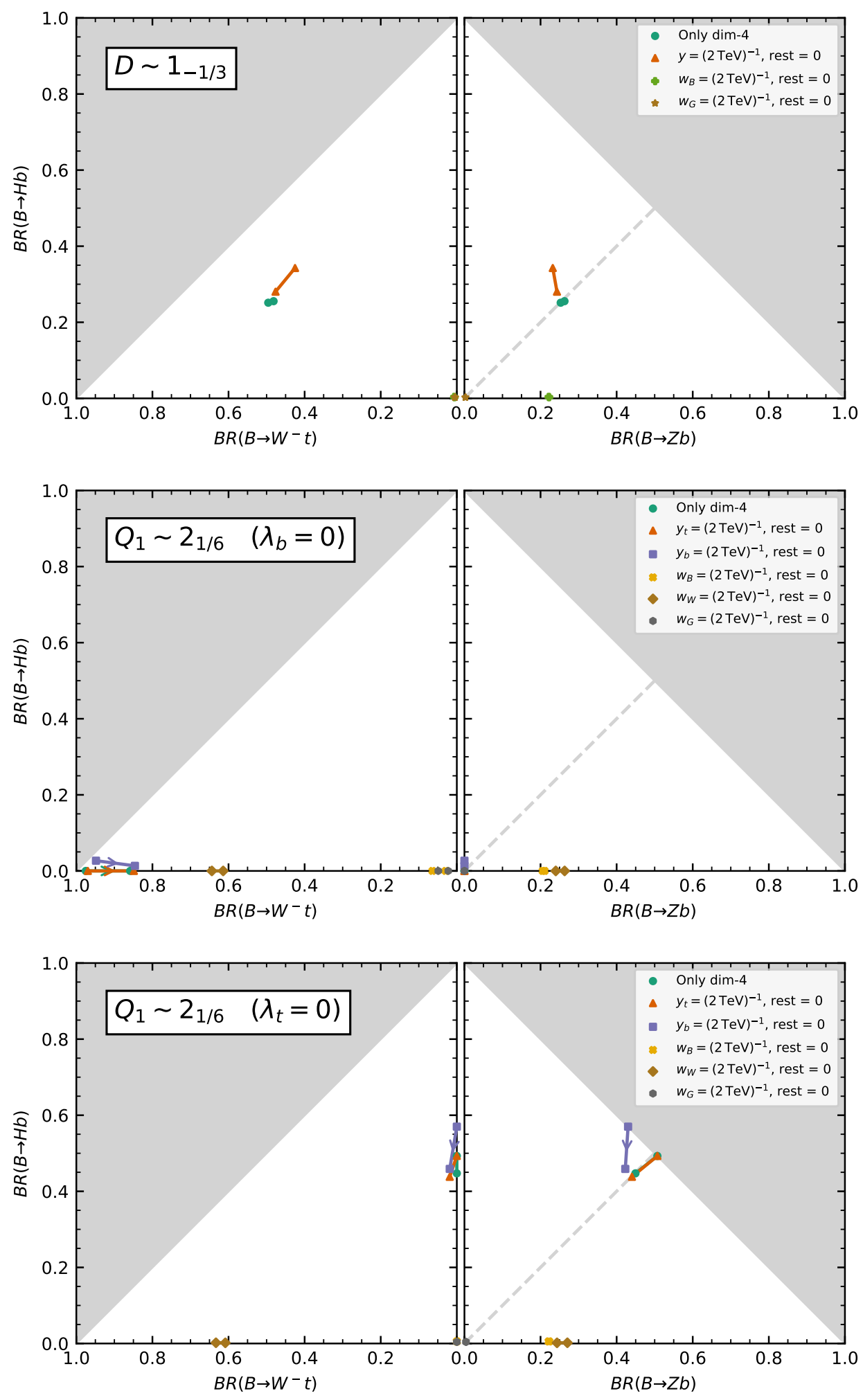

Figure 12. Branching ratios of $B$ into $H b, Z b$ and $W^{-} t$ for various values of the parameters in the $D$ and $Q_{1}$ models. The dimensionless couplings $\lambda$ are always chosen to saturate the corresponding electroweak precision bounds. 

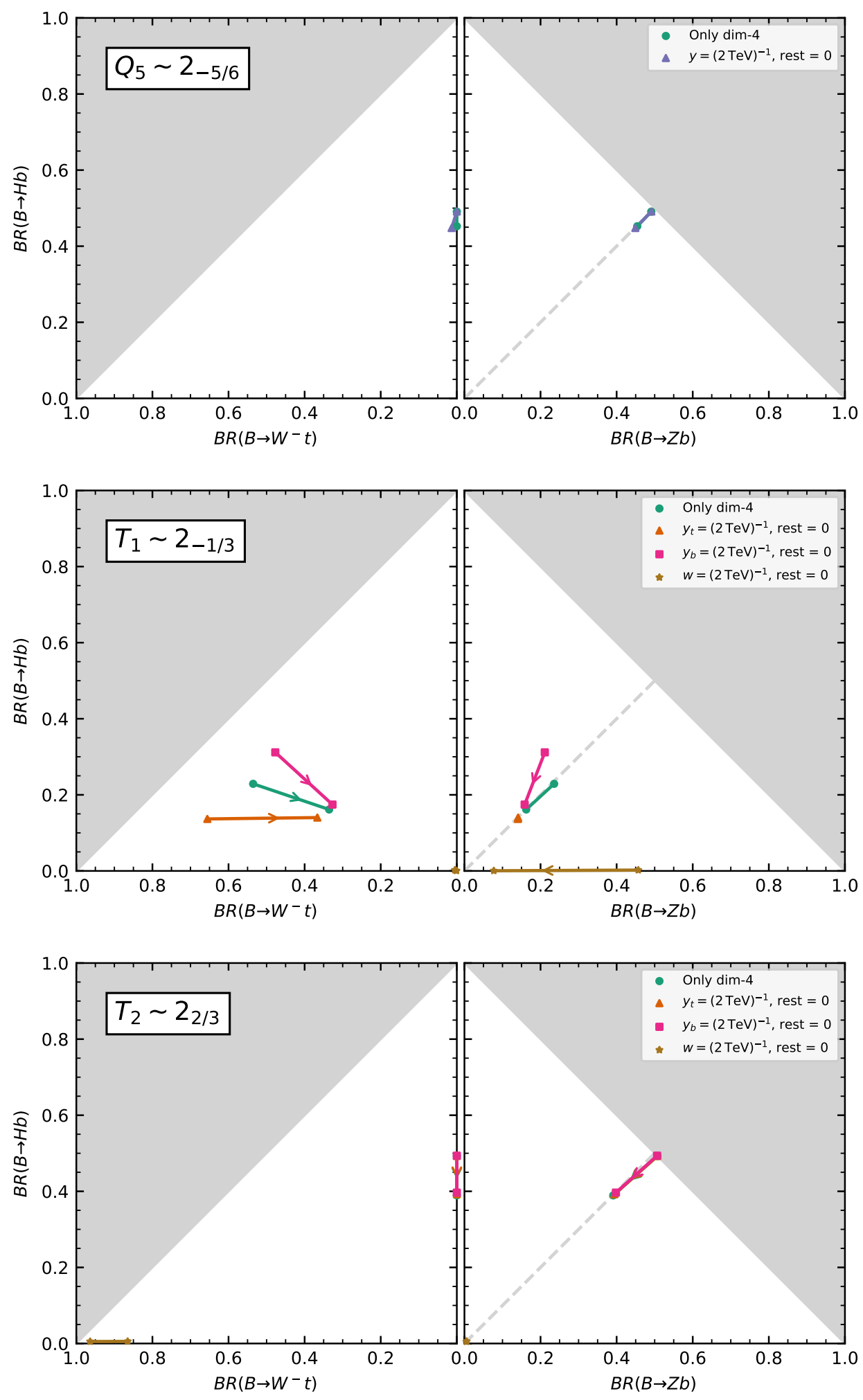

Figure 13. Branching ratios of $B$ into $H b, Z b$ and $W^{-} t$ for various values of the parameters in the $Q_{5}, T_{2}$ and $T_{1}$ models. The dimensionless couplings $\lambda$ are always chosen to saturate the corresponding electroweak precision bounds. 

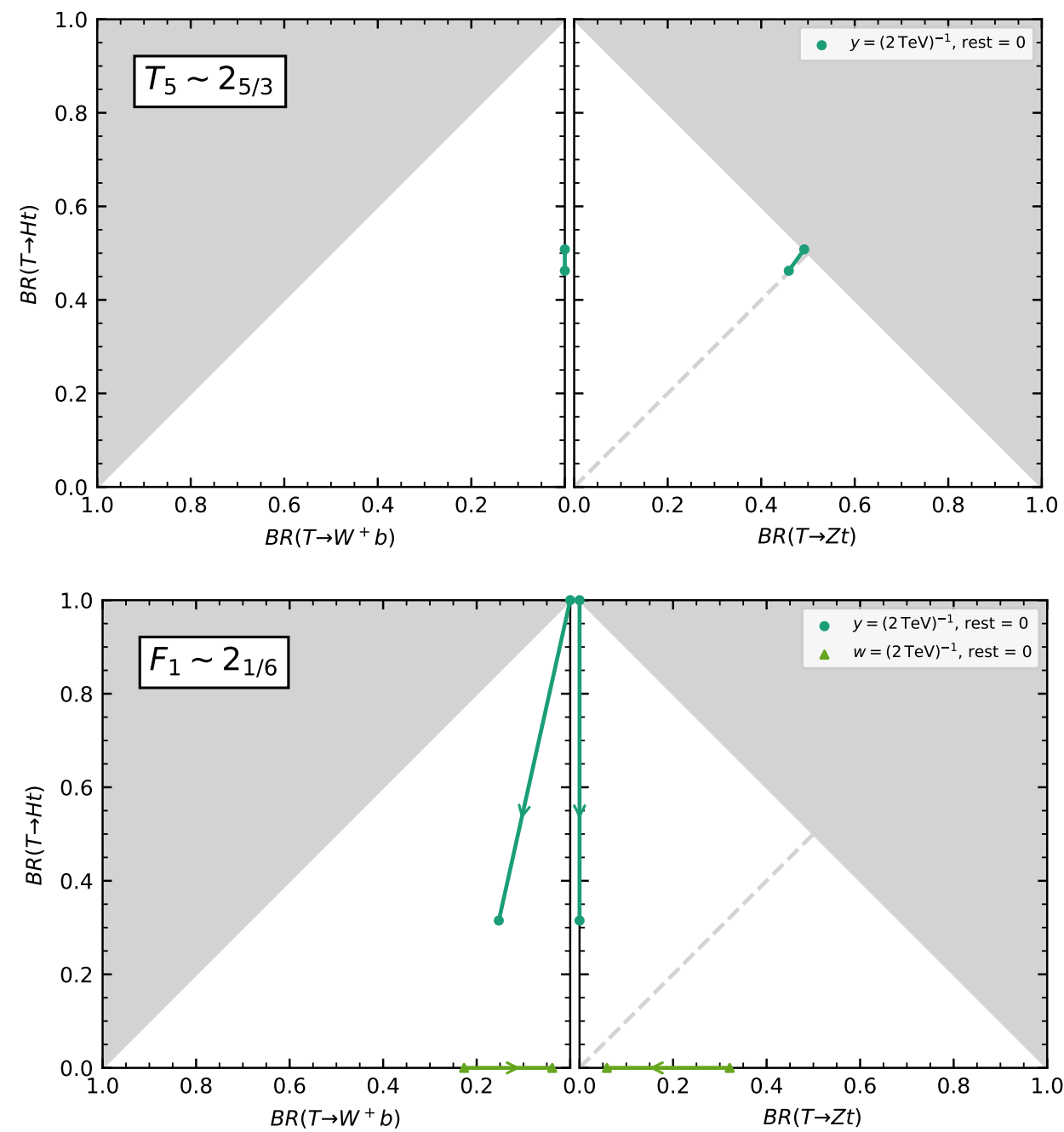

Figure 14. Branching ratios of $T$ into $H t, Z t$ and $W^{+} b$ for various values of the parameters in the $T_{5}$ and $F_{7}$ models. 

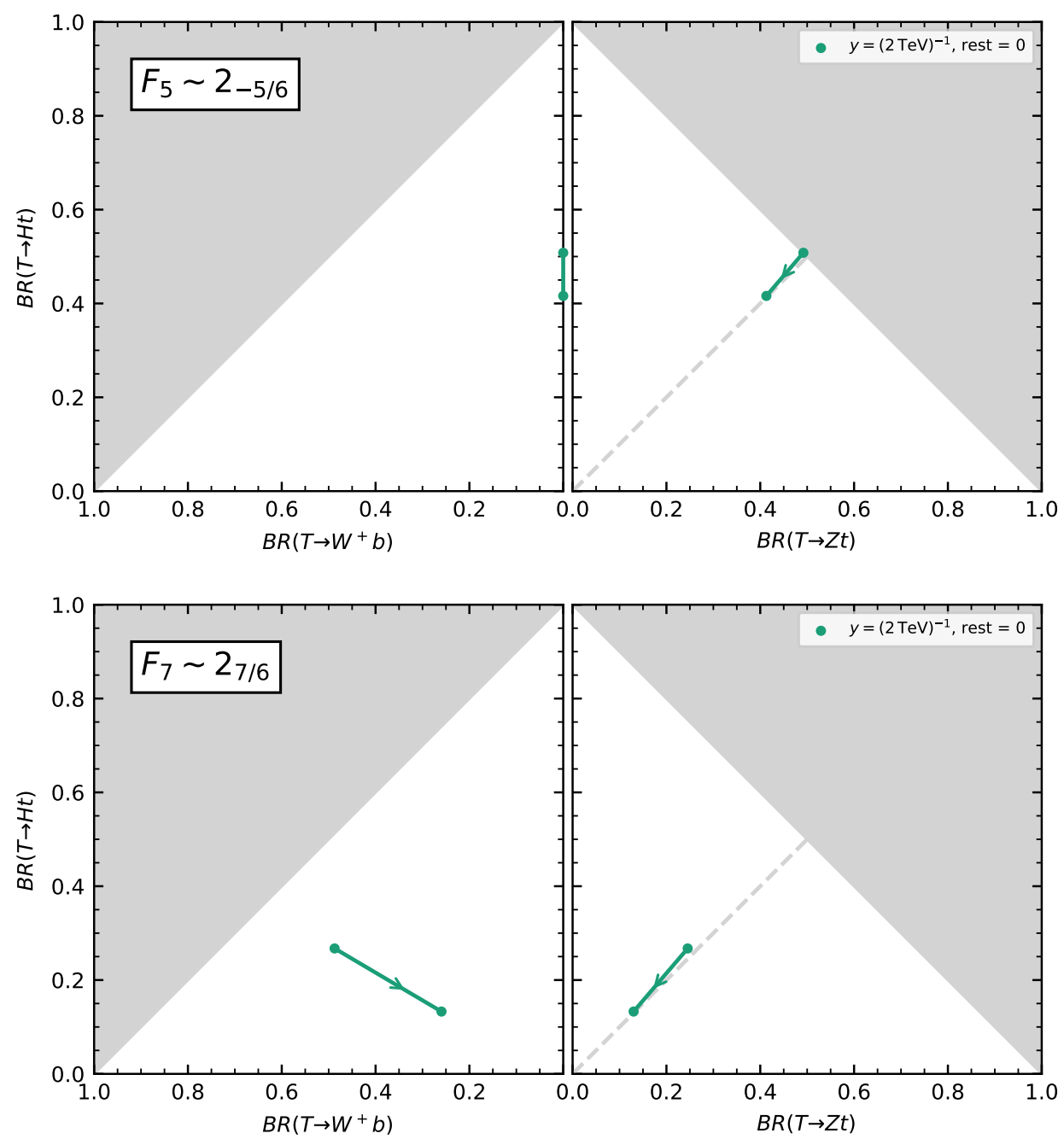

Figure 15. Branching ratios of $T$ into $H t, Z t$ and $W^{+} b$ for various values of the parameters in the $F_{1}$ and $F_{5}$ models. 

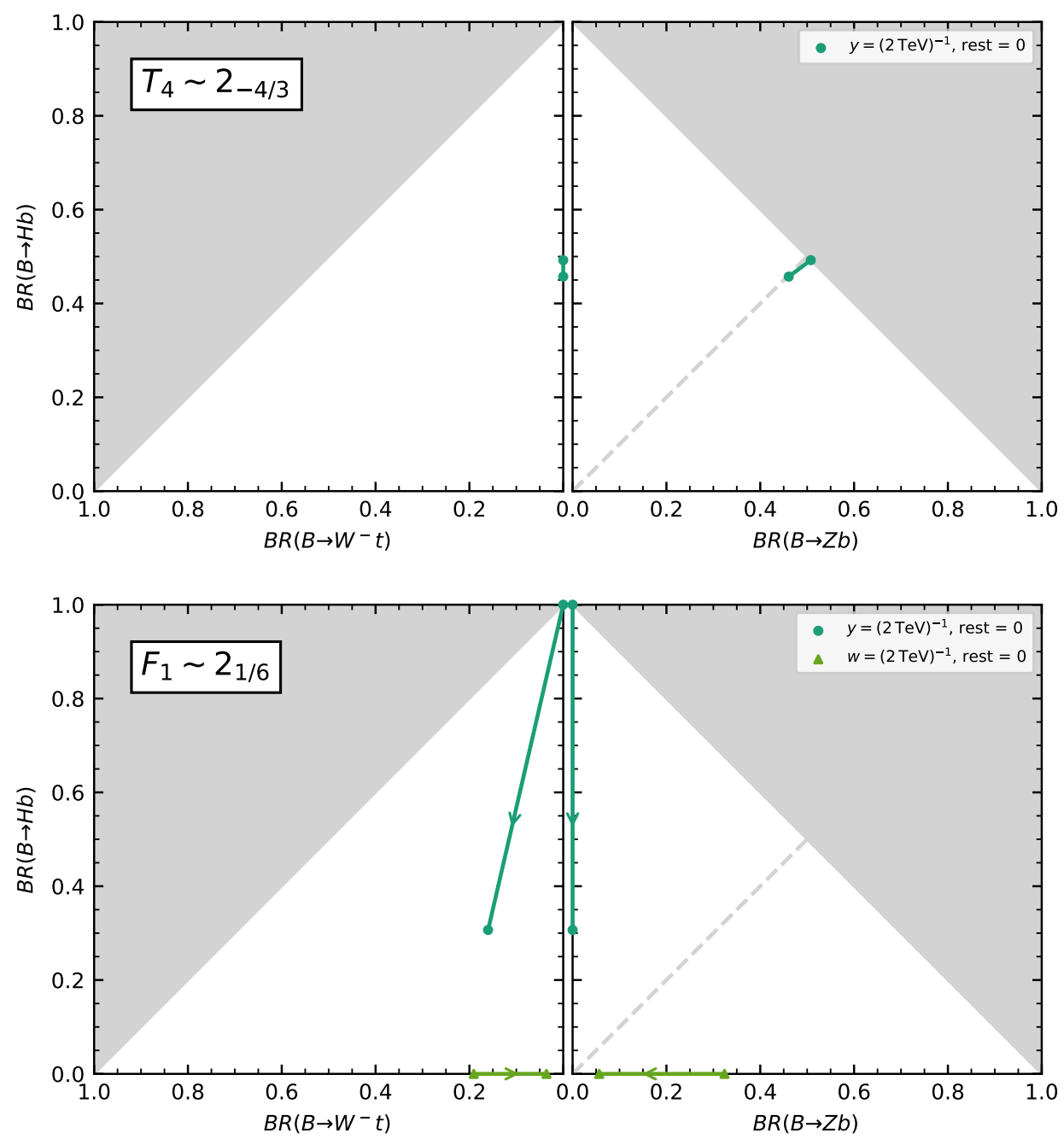

Figure 16. Branching ratios of $B$ into $H b, Z b$ and $W^{-} t$ for various values of the parameters in the $T_{4}$ and $F_{7}$ models. 

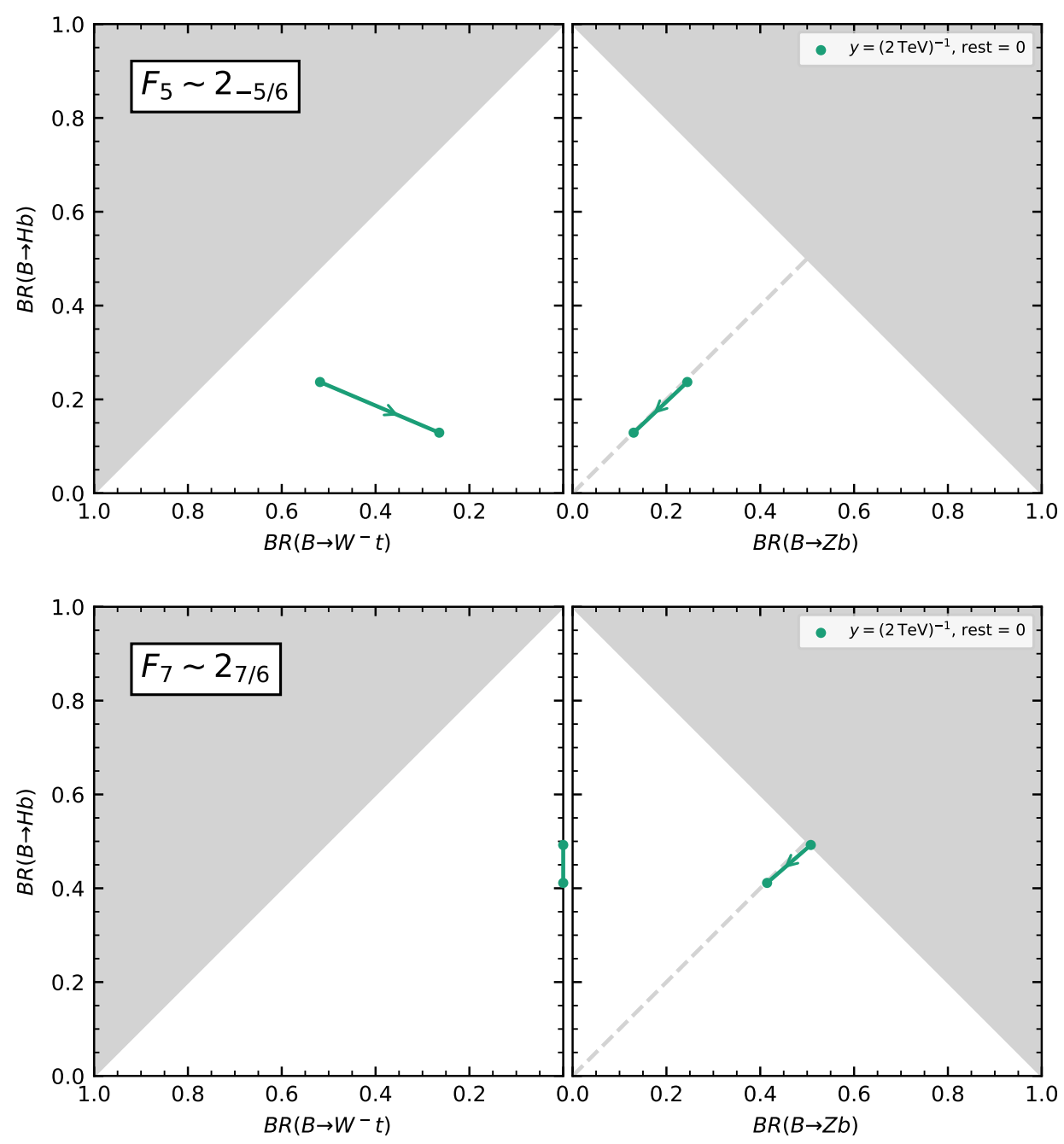

Figure 17. Branching ratios of $T$ into $H t, Z t$ and $W^{-} t$ for various values of the parameters in the $F_{1}$ and $F_{5}$ models.

\section{Conclusions}

The phenomenology of vector-like extra quarks near the TeV scale is to a large extent governed by gauge invariance and power counting. To start with, extra quarks can always be pair produced at hadron colliders by their gauge coupling to gluons. Once produced, they will decay into Standard Model particles if they have gauge-invariant linear interactions with them. At the renormalizable level, this is only possible for seven different gaugecovariant multiplets. These are the multiplets that can have Yukawa couplings with the Higgs doublet, which mix the extra quarks among themselves and with the SM ones. The latter mixing gives rise to decays into a SM quark and either $Z, W$ or Higgs bosons. In simple extensions with only one vector-like multiplet, these are the only significant decay modes. Furthermore, in the motivated case of exclusive mixing with the third generation, the branching ratios are fixed by the quantum numbers of the multiplet. The mixing is also responsible for indirect effects, mass splittings and single production. 
This simple picture can be modified in three ways (or combinations of them). First, one can consider general couplings to all the three SM generations [13, 61-64]. This typically requires flavour symmetries to evade the strong flavour constraints. Sizable mixing with the valence quarks in the proton would increase the importance of single production [63]. Second, it is possible to consider several vector-like quark multiplets, or other additional particles, like scalars or vector bosons. This may give rise to new production mechanisms [65] or new decay modes $[33,59,66]$, in addition to the standard ones described above. Third, one can drop the assumption of renormalizability. This is the path we have explored in this work. We have proposed a model-independent approach that uses a general local effective Lagrangian, valid up to a cutoff scale $\Lambda$ and constructed with the SM fields and the fields that represent arbitrary new vector-like quarks. This is a faithful description of any model with new vector-like quarks, as long as the new physics not explicitly included appears at scales higher than $\Lambda$. In particular, the effective theory describes well the case of additional particles when they are heavier than $\Lambda$. As usual, the effective Lagrangian is defined by its expansion in inverse powers of $\Lambda$. The lowest order, formed by operators of canonical dimension $\leq 4$, corresponds to the usual renormalizable theories with extra vector-like quarks. The interactions of higher dimension give contributions to observables suppressed by powers of $\mu / \Lambda$, with $\mu=E, M, v$ the characteristic scale of the process. Even if suppressed, these interactions can be very relevant for proceses that do not exist at the renormalizable level.

In our explicit phenomenological analysis we have worked with the effective theory for extensions of the SM with only one vector-like quark multiplet and we have truncated it at the next-to-leading order, i.e. at canonical dimension 5. For simplicity we have also assumed couplings to the third generation only. At this order, there are twelve irreducible representations of extra quarks that can decay into SM particles (and be singly produced). Up to field redefinition ambiguities, four new types of interactions appear at dimension 5 : the Yukawa-type operators $\bar{Q} q \phi \phi$ and $\bar{Q} Q \phi \phi$ and the "magnetic" operators $\bar{Q} \sigma^{\mu \nu} q F_{\mu \nu}$ and $\bar{Q} \sigma_{\mu \nu} Q F_{\mu \nu}$. The latter have effects that increase with the energy of the process.

We have distinguished two types of vector-like quarks. Those in the seven representations that allow for renormalizable linear interactions, and those in the remaining five representations. For the extra quark in the first group, and for natural values of the coupling constants, the dimension-5 interactions typically give only small corrections to the standard phenomenology of vector-like quarks. One exception is the possibility of new indirect effects in Higgs physics. Moreover, in strongly-coupled UV completions avoiding the loop suppression in the "magnetic" couplings, there can be new single production modes with cross sections larger than the one of pair production and also new decay modes (into $q g$, for instance) with large branching ratios. Of course, all these effects depend on the cutoff and will be negligible if $\Lambda$ is much larger than the TeV scale.

For the quarks in the five multiplets that do not have renormalizable linear interactions (two triplets and four quadruplets), the dimension-5 operators give the leading contributions. In this case, all the indirect bounds can be easily evaded without explicit tuning of couplings, for moderate values of $\Lambda$. Pair production is still possible and the decay (possibly after hadronization) will be prompt if $\Lambda$ is not too high. Some non-standard 
decay modes, including three-body decays, can be sizable and the measurements of decays of $T$ and $B$ into $Z q, W q$ and $H q$ could easily give rise to new points in the corresponding triangles. In this respect, we have given a simple formula to recast the combination limits given by the ATLAS and CMS collaborations, which assume the absence of other decay channels. For $\Lambda \gtrsim 10^{6} \mathrm{TeV}$, the decays of the hadrons containing the heavy quarks will be non-prompt. The usual searches will not be sensitive to vector-like quarks in this regime, but one can instead resort to the signatures associated to coloured and charged long-lived particles. Taking advantage of these signatures would require dedicated searches of vectorlike quarks, specially in the case of displaced vertices formed by their decay products.

New operators involving the extra quarks appear at yet higher orders in the $1 / \Lambda$ expansion. At dimension 6 one should include four fermion operators [67]. In particular, the interactions of the form $q q q Q$ will give rise to new single production mechanisms, which can have observable cross sections at the LHC for $\Lambda$ of a few TeV when the couplings to the first generation are allowed. Moreover, at each order new types of vector-like quarks will be able to decay into SM particles. Their lifetime will be suppressed by the corresponding power of $M / \Lambda$. Finally, in principle it is also possible that new vector-like quarks exist in gauge representations with $T+Y+1 / 3 \notin \mathbb{Z}$. They would be stable or else decay into additional stable particles. However, there are very strong constraints on the abundances of stable strongly interacting (and charged) particles, in particular from searches of rare nuclei $[68-72] .^{8}$

\section{Acknowledgments}

We thank Juan Antonio Aguilar Saavedra, Miki Chala and Michelangelo Mangano for useful discussions. Our work has been supported by the Spanish MINECO project FPA201678220-C3-1-P (Fondos FEDER) and the Junta de Andalucía grant FQM101. The work of J.C.C. has also been supported by the Spanish MECD grant FPU14.

\section{A Representations of Standard Model operators}

In this section, we obtain a constraint over the representation of any operator constructed as a product of the Standard Model fields. A representation of $\mathrm{SU}(3) \times \mathrm{SU}(2) \times \mathrm{U}(1)$ is denoted by $(\rho, \sigma, Y)$, where $\rho$ is the representation under $\mathrm{SU}(3), \sigma$ is the representation under $\mathrm{SU}(2)$ and $Y$ is the hypercharge. We define

$$
N(\rho, \sigma, Y)=A(\rho)+B(\sigma)+Y,
$$

with $0 \leq A(\rho), B(\sigma)<1$ satisfying the equations

$$
\rho\left(e^{2 i \pi / 3} I\right)=e^{2 i \pi A(\rho)} I, \quad \sigma(-I)=e^{2 i \pi B(\sigma)} I .
$$

\footnotetext{
${ }^{8}$ See, nevertheless, ref. [73] for comments on the robustness of such bounds and a proposal of coloured dark matter.
} 
The values $A$ and $B$ may take are limited: $A(\rho) \in\{0,1 / 3,2 / 3\}$ and $B(\sigma) \in\{0,1 / 2\}$. The set representations of $\mathrm{SU}(3)$ is split into three classes by $A$ :

$$
\begin{aligned}
0 & =A(1)=A(8)=A(10)=A(\overline{10})=A(27)=\ldots \\
1 / 3 & =A(3)=A(\overline{6})=A(15)=A\left(15^{\prime}\right)=A(24)=\ldots \\
2 / 3 & =A(\overline{3})=A(6)=A(\overline{15})=A\left(\overline{15^{\prime}}\right)=A(\overline{24})=\ldots
\end{aligned}
$$

while $B$ splits the set of $\mathrm{SU}(2)$ representations in two: those with integer spin and the others. Both $A$ and $B$ are additive under the operation of taking tensor products of representations:

$$
\begin{aligned}
& A\left(\rho_{1} \otimes \rho_{2}\right)=A\left(\rho_{1}\right)+A\left(\rho_{2}\right)(\bmod 1), \\
& B\left(\sigma_{1} \otimes \sigma_{2}\right)=B\left(\sigma_{1}\right)+B\left(\sigma_{2}\right)(\bmod 1) .
\end{aligned}
$$

We will prove now that if $\left(\rho_{\mathcal{O}}, \sigma_{\mathcal{O}}, Y_{\mathcal{O}}\right)$ is the representation of some operator $\mathcal{O}$ constructed with the Standard Model fields, then $N\left(\rho_{\mathcal{O}}, \sigma_{\mathcal{O}}, Y_{\mathcal{O}}\right)$ is an integer. First, it can be directly checked that $N\left(\rho_{\phi}, \sigma_{\phi}, Y_{\phi}\right)$ is an integer for any Standard Model field $\phi$. Now, from the additivity of $A, B$ and $Y$ it follows that the value of $N$ corresponding to the product $\mathcal{O Q}$ of two operators $\mathcal{O}$ and $\mathcal{Q}$ is

$$
\begin{aligned}
N\left(\rho_{\mathcal{O} \mathcal{Q}}, \sigma_{\mathcal{O} \mathcal{Q}}, Y_{\mathcal{O Q}}\right) & =\left[A\left(\rho_{\mathcal{O}}\right)+A\left(\rho_{\mathcal{Q}}\right)\right]+\left[B\left(\sigma_{\mathcal{O}}\right)+B\left(\sigma_{\mathcal{Q}}\right)\right]+\left[Y_{\mathcal{O}}+Y_{\mathcal{Q}}\right](\bmod 1) \\
& =\left[A\left(\rho_{\mathcal{O}}\right)+B\left(\sigma_{\mathcal{O}}\right)+Y_{\mathcal{O}}\right]+\left[A\left(\rho_{\mathcal{Q}}\right)+B\left(\sigma_{\mathcal{Q}}\right)+Y_{\mathcal{Q}}\right](\bmod 1) \\
& =N\left(\rho_{\mathcal{O}}, \sigma_{\mathcal{O}}, Y_{\mathcal{O}}\right)+N\left(\rho_{\mathcal{Q}}, \sigma_{\mathcal{Q}}, Y_{\mathcal{Q}}\right)(\bmod 1),
\end{aligned}
$$

Therefore, if $N\left(\rho_{\mathcal{O}}, \sigma_{\mathcal{O}}, Y_{\mathcal{O}}\right)$ and $N\left(\rho_{\mathcal{Q}}, \sigma_{\mathcal{Q}}, Y_{\mathcal{Q}}\right)$ are integers, then $N\left(\rho_{\mathcal{O} \mathcal{Q}}, \sigma_{\mathcal{O} \mathcal{Q}}, Y_{\mathcal{O Q}}\right)$ must also be integer. This completes the proof.

Particularizing for SU(3) triplets, we get

$$
T+Y+1 / 3 \in \mathbb{Z}
$$

where $T$ is the spin of the $\mathrm{SU}(2)$ representation.

\section{B Limits on the mass for the case with extra decays}

Experimental data determines an upper limit $L_{\exp }$ on the sum of the cross-sections for the production and decay of a pair of heavy quarks, weighted by the efficiency for each decay channel (see ref. [33]):

$$
\sigma_{p p \rightarrow Q \bar{Q}}(M) \sum_{i j} \epsilon_{i j} B R_{i} B R_{j}<L_{\exp },
$$

where $M$ is the mass of the heavy quark, $i$ and $j$ run over all the decay channels, and $\epsilon_{i j}$ is the corresponding efficiency. A limit on the mass can be derived from this inequality. In the usual experimental analyses, it is assumed that the sum of the branching ratios into these three channels is $\Sigma=1$.

We consider now the case $\Sigma<1$. We will obtain a lower limit on the mass of some heavy quark with branching ratios $B R_{i}$. Some assumption has to be made about the 
efficiency $\epsilon_{i a}=\epsilon_{a i}$ for the channels $a$ that are not $H q, Z q$ or $W^{ \pm} q^{\prime}$. We adopt here the conservative choice $\epsilon_{i a}=0$. Let $M_{1}$ be the lower limit on the mass for the branching ratios $B R_{i}^{\Sigma}=B R_{i} / \Sigma$, whose sum is 1 , so that $M_{1}$ is known from experimental analyses. We define the mass $M_{\Sigma}$ by the equation

$$
\Sigma^{2} \sigma_{p p \rightarrow Q \bar{Q}}\left(M_{\Sigma}\right)=\sigma_{p p \rightarrow Q \bar{Q}}\left(M_{1}\right) .
$$

Then, we have the identity

$$
\sigma_{p p \rightarrow Q \bar{Q}}\left(M_{\Sigma}\right) \sum_{i j} \epsilon_{i j} B R_{i} B R_{j}=\sigma_{p p \rightarrow Q \bar{Q}}\left(M_{1}\right) \sum_{i j} \epsilon_{i j} B R_{i}^{\Sigma} B R_{j}^{\Sigma} .
$$

Because $M_{1}$ is the limit obtained from eq. (B.1) for branching ratios $B R_{i}^{\Sigma}$, it follows from this identity that $M_{\Sigma}$ is the limit for $B R_{i}$. We now proceed to find an analytic solution to eq. (B.2). The production cross-section $\sigma_{p p \rightarrow Q \bar{Q}}(M)$ can be approximated, for masses around $\widetilde{M}=1.1 \mathrm{TeV}$ by an exponential:

$$
\sigma_{p p \rightarrow Q \bar{Q}}(M) \simeq \sigma_{p p \rightarrow Q \bar{Q}}(\widetilde{M}) \exp \left(-\frac{M^{1 / 2}-\widetilde{M}^{1 / 2}}{f^{1 / 2} / 2}\right),
$$

where $f=20.5 \mathrm{GeV}$. In the range $[0.8,1.4] \mathrm{TeV}$, the difference between the cross section produced by this formula and the one obtained using MadGraph increases towards the extremes of the interval and is at most $3 \%$. Plugging eq. (B.4) in eq. (B.2) gives

$$
M_{\Sigma}=\left(M_{1}^{1 / 2}+f^{1 / 2} \log \Sigma\right)^{2} .
$$

\section{Approximate equality of the branching ratios to $H q$ and $Z q$}

We provide here an explanation for the fact that the branching ratios of a heavy quark $Q=T, B$ with only Yukawa-type couplings $(Q \phi q$ and $Q \phi \phi q)$ into $Z t$ and $H t$ are approximately equal, for $Q$ in any multiplet except $F_{1}$. We define $X_{Q q}^{L, R}$ and $Y_{Q q}^{L, R}$ as the following coefficients in the Lagrangian:

$$
\begin{aligned}
\mathcal{L}_{Z} & =-\frac{g}{2 c_{W}} \bar{q} \text { 虫 }\left( \pm X_{q Q}^{L} P_{L} \pm X_{q Q}^{R} P_{R}\right) Q+\text { h.c. }, \\
\mathcal{L}_{H} & =-\frac{g m_{Q}}{2 m_{W}} \bar{q} H\left(Y_{q Q}^{L} P_{L}+Y_{q Q}^{R} P_{R}\right) Q,
\end{aligned}
$$

the equality of the braching ratios follows from the equality in magnitude of the dominant $X_{Q q}^{L, R}$ and the dominant $Y_{Q q}^{L, R}$.

The weak eigenstates $q^{0}, Q^{0}$ couple to the $Z$ boson as

$$
\mathcal{L}_{Z}=-\frac{g}{2 c_{W}} \sum_{\chi=L, R}\left(\begin{array}{ll}
\bar{q}_{\chi}^{0} & \bar{Q}_{\chi}^{0}
\end{array}\right) \not\left(\begin{array}{cc}
2 T_{3}\left(q_{\chi}^{0}\right)-2 Q_{e}\left(q_{\chi}^{0}\right) s_{W}^{2} & 0 \\
0 & 2 T_{3}\left(Q_{\chi}^{0}\right)-2 Q_{e}\left(Q_{\chi}^{0}\right) s_{W}^{2}
\end{array}\right)\left(\begin{array}{c}
q_{\chi}^{0} \\
Q_{\chi}^{0}
\end{array}\right),
$$

where $T_{3}$ denotes the third component of isospin and $Q_{e}$ denotes electric charge. After the unitary transformation in equations (3.9) and (3.10), we get

$$
X_{q Q}^{L, R}=2 s_{L, R} c_{L, R}\left[T_{3}\left(q_{L, R}^{0}\right)-T_{3}\left(Q_{L, R}^{0}\right)\right] .
$$


On the other hand, the quark gauge eigenstates $q^{0}, Q^{0}$ couple to the Higgs as

$$
\mathcal{L}_{H}=-\frac{1}{\sqrt{2}}\left(\begin{array}{cc}
\bar{q}_{L}^{0} & \bar{Q}_{L}^{0}
\end{array}\right) H\left(\begin{array}{cc}
y_{11} & y_{12} \\
y_{21} & 0
\end{array}\right)\left(\begin{array}{c}
q_{R}^{0} \\
Q_{R}^{0}
\end{array}\right) .
$$

Generally, one of the off-diagonal elements is negligible. This happens because the dimension- 4 and dimension-5 Yukawas always contribute to different elements of the $y_{i j}$ matrix. Either one of them is zero or, when both are present, the dimension- 5 one is smaller. This means that one of the mixing angles $\theta_{L, R}$ dominates. For multiplets with dimension4 couplings, the chirality with the dominant mixing angle $\theta_{D}$ is $D=L$ for singlets and triplets and $D=R$ for doublets. For multiplets without dimension- 4 couplings it is $D=R$ for triplets and $D=L$ for quadruplets.

The dominant off-diagonal element $y_{D}$ is related to the corresponding mixing angle as $y_{D} \simeq x \sqrt{2} m_{Q} s_{D} / v$, where $x=1$ in the cases with dimension- 4 interactions and $x=2$ in the ones with only dimension- 5 ones. This factor is necessary because of the different the relation between the mass and Yukawa terms in both cases. The dominant $H q Q$ coupling is, then

$$
Y_{Q q}^{D} \simeq x s_{D} c_{D}
$$

For $X_{Q q}^{D} \simeq Y_{Q q}^{D}$ it is necessary and sufficient that

$$
\left|T_{3}\left(q_{D}^{0}\right)-T_{3}\left(Q_{D}^{0}\right)\right|=x / 2 .
$$

It can be checked case by case that this relation is statisfied for all multiplets except for $F_{1}$. In this case, we have $\left|T_{3}\left(q_{L}^{0}\right)-T_{3}\left(Q_{L}^{0}\right)\right|=0$.

Open Access. This article is distributed under the terms of the Creative Commons Attribution License (CC-BY 4.0), which permits any use, distribution and reproduction in any medium, provided the original author(s) and source are credited.

\section{References}

[1] F. del Aguila, J. de Blas and M. Pérez-Victoria, Effects of new leptons in electroweak precision data, Phys. Rev. D 78 (2008) 013010 [arXiv:0803.4008] [INSPIRE].

[2] F. del Aguila and J.A. Aguilar-Saavedra, Distinguishing seesaw models at LHC with multi-lepton signals, Nucl. Phys. B 813 (2009) 22 [arXiv:0808.2468] [INSPIRE].

[3] H. Fritzsch, M. Gell-Mann and P. Minkowski, Vector-like weak currents and new elementary fermions, Phys. Lett. B 59 (1975) 256.

[4] J. Kearney, A. Pierce and J. Thaler, Exotic top partners and little Higgs, JHEP 10 (2013) 230 [arXiv: 1306.4314] [INSPIRE].

[5] K. Agashe, R. Contino and A. Pomarol, The minimal composite Higgs model, Nucl. Phys. B 719 (2005) 165 [hep-ph/0412089] [inSPIRE].

[6] P. Batra, B.A. Dobrescu and D. Spivak, Anomaly-free sets of fermions, J. Math. Phys. 47 (2006) 082301 [hep-ph/0510181] [INSPIRE]. 
[7] D.B. Kaplan, Flavor at SSC energies: a new mechanism for dynamically generated fermion masses, Nucl. Phys. B 365 (1991) 259 [InSPIRE].

[8] T. Gherghetta and A. Pomarol, Bulk fields and supersymmetry in a slice of AdS, Nucl. Phys. B 586 (2000) 141 [hep-ph/0003129] [INSPIRE].

[9] D. Choudhury, T.M.P. Tait and C.E.M. Wagner, Beautiful mirrors and precision electroweak data, Phys. Rev. D 65 (2002) 053002 [hep-ph/0109097] [INSPIRE].

[10] N. Arkani-Hamed, A.G. Cohen and H. Georgi, Electroweak symmetry breaking from dimensional deconstruction, Phys. Lett. B 513 (2001) 232 [hep-ph/0105239] [INSPIRE].

[11] R. Contino, L. Da Rold and A. Pomarol, Light custodians in natural composite Higgs models, Phys. Rev. D 75 (2007) 055014 [hep-ph/0612048] [InSPIRE].

[12] F. del Aguila and M.J. Bowick, The possibility of new fermions with $\Delta I=0$ mass, Nucl. Phys. B 224 (1983) 107 [INSPIRE].

[13] F. del Aguila, M. Pérez-Victoria and J. Santiago, Observable contributions of new exotic quarks to quark mixing, JHEP 09 (2000) 011 [hep-ph/0007316] [INSPIRE].

[14] F. del Aguila, M. Pérez-Victoria and J. Santiago, Effective description of quark mixing, Phys. Lett. B 492 (2000) 98 [hep-ph/0007160] [INSPIRE].

[15] L. Lavoura and J.P. Silva, The oblique corrections from vector-like singlet and doublet quarks, Phys. Rev. D 47 (1993) 2046 [InSPIRE].

[16] M. Carena, E. Ponton, J. Santiago and C.E.M. Wagner, Light Kaluza Klein states in Randall-Sundrum models with custodial SU(2), Nucl. Phys. B 759 (2006) 202 [hep-ph/0607106] [INSPIRE].

[17] C. Anastasiou, E. Furlan and J. Santiago, Realistic composite Higgs models, Phys. Rev. D 79 (2009) 075003 [arXiv:0901.2117] [INSPIRE].

[18] J.A. Aguilar-Saavedra, Identifying top partners at LHC, JHEP 11 (2009) 030 [arXiv:0907.3155] [INSPIRE].

[19] J.A. Aguilar-Saavedra, R. Benbrik, S. Heinemeyer and M. Pérez-Victoria, Handbook of vectorlike quarks: mixing and single production, Phys. Rev. D 88 (2013) 094010 [arXiv:1306.0572] [INSPIRE].

[20] G. Cacciapaglia, A. Deandrea, D. Harada and Y. Okada, Bounds and decays of new heavy vector-like top partners, JHEP 11 (2010) 159 [arXiv:1007.2933] [INSPIRE].

[21] S. Beauceron, G. Cacciapaglia, A. Deandrea and J.D. Ruiz-Alvarez, Fully hadronic decays of a singly produced vectorlike top partner at the LHC, Phys. Rev. D 90 (2014) 115008 [arXiv: 1401.5979] [INSPIRE].

[22] D. Barducci et al., Framework for model independent analyses of multiple extra quark scenarios, JHEP 12 (2014) 080 [arXiv:1405.0737] [INSPIRE].

[23] S. Moretti, D. O'Brien, L. Panizzi and H. Prager, Production of extra quarks at the Large Hadron Collider beyond the narrow width approximation, Phys. Rev. D 96 (2017) 075035 [arXiv: 1603.09237] [INSPIRE].

[24] A. Carvalho et al., Single production of vectorlike quarks with large width at the Large Hadron Collider, Phys. Rev. D 98 (2018) 015029 [arXiv:1805.06402] [INSPIRE].

[25] O. Matsedonskyi, G. Panico and A. Wulzer, Light top partners for a light composite Higgs, JHEP 01 (2013) 164 [arXiv:1204.6333] [INSPIRE]. 
[26] A. De Simone, O. Matsedonskyi, R. Rattazzi and A. Wulzer, A first top partner hunter's guide, JHEP 04 (2013) 004 [arXiv: 1211.5663] [INSPIRE].

[27] O. Matsedonskyi, G. Panico and A. Wulzer, Top partners searches and composite Higgs models, JHEP 04 (2016) 003 [arXiv:1512.04356] [INSPIRE].

[28] R. Rattazzi, Cargese lectures on extra dimensions, in the proceedings of Particle physics and cosmology: the interface, August 4-16, Cargese, France (2003) [CERN-PH-TH-2006-029] [hep-ph/0607055].

[29] S. Fajfer, A. Greljo, J.F. Kamenik and I. Mustac, Light Higgs and vector-like quarks without prejudice, JHEP 07 (2013) 155 [arXiv:1304.4219] [INSPIRE].

[30] A. Alloul et al., FeynRules 2.0 - A complete toolbox for tree-level phenomenology, Comput. Phys. Commun. 185 (2014) 2250 [arXiv:1310.1921] [INSPIRE].

[31] J. Alwall et al., MadGraph 5: going beyond, JHEP 06 (2011) 128 [arXiv:1106.0522] [INSPIRE].

[32] J. Alwall et al., Computing decay rates for new physics theories with FeynRules and MadGraph 5_aMC@NLO, Comput.Phys.Commun. 197 (2015) 312 [arXiv:1402.1178] [INSPIRE].

[33] J.A. Aguilar-Saavedra, D.E. López-Fogliani and C. Muñoz, Novel signatures for vector-like quarks, JHEP 06 (2017) 095 [arXiv:1705. 02526] [INSPIRE].

[34] Y. Kats and M.J. Strassler, Probing colored particles with photons, leptons and jets, JHEP 11 (2012) 097 [Erratum ibid. 1607 (2016) 009] [arXiv: 1204.1119] [INSPIRE].

[35] J.H. Kim and I.M. Lewis, Loop induced single top partner production and decay at the LHC, JHEP 05 (2018) 095 [arXiv: 1803.06351] [INSPIRE].

[36] S. Dawson and E. Furlan, A Higgs conundrum with vector fermions, Phys. Rev. D 86 (2012) 015021 [arXiv: 1205.4733] [INSPIRE].

[37] J. de Blas, J.C. Criado, M. Pérez-Victoria and J. Santiago, Effective description of general extensions of the Standard Model: the complete tree-level dictionary, JHEP 03 (2018) 109 [arXiv: 1711.10391] [INSPIRE].

[38] J.A. Aguilar-Saavedra, A minimal set of top-Higgs anomalous couplings, Nucl. Phys. B 821 (2009) 215 [arXiv:0904.2387] [INSPIRE].

[39] B. Grzadkowski, M. Iskrzynski, M. Misiak and J. Rosiek, Dimension-six terms in the standard model lagrangian, JHEP 10 (2010) 085 [arXiv:1008.4884] [INSPIRE].

[40] ATLAS collaboration, Observation of Higgs boson production in association with a top quark pair at the LHC with the ATLAS detector, Phys. Lett. B 784 (2018) 173 [arXiv: 1806.00425] [INSPIRE].

[41] CMS collaboration, Observation of t $\bar{t} H$ production, Phys. Rev. Lett. 120 (2018) 231801 [arXiv: 1804.02610] [INSPIRE].

[42] M. Cepeda et al., Report from Working Group 2, CERN Yellow Rep. Monogr. 7 (2019) 221 [arXiv: 1902.00134] [INSPIRE].

[43] J. Ellis, C.W. Murphy, V. Sanz and T. You, Updated global SMEFT fit to Higgs, diboson and electroweak data, JHEP 06 (2018) 146 [arXiv:1803.03252] [INSPIRE]. 
[44] ATLAS, CMS collaboration, Addendum to the report on the physics at the HL-LHC and perspectives for the HE-LHC: Collection of notes from ATLAS and CMS, CERN Yellow Rep. Monogr. 7 (2019) Addendum [arXiv: 1902.10229] [INSPIRE].

[45] J.A. Aguilar-Saavedra, Effective four-fermion operators in top physics: A Roadmap, Nucl. Phys. B 843 (2011) 638 [Erratum ibid. B 851 (2011) 443] [arXiv:1008.3562] [INSPIRE].

[46] A. Buckley et al., Constraining top quark effective theory in the LHC Run II era, JHEP 04 (2016) 015 [arXiv: 1512.03360] [INSPIRE].

[47] V. Cirigliano, W. Dekens, J. de Vries and E. Mereghetti, Is there room for CP-violation in the top-Higgs sector?, Phys. Rev. D 94 (2016) 016002 [arXiv:1603.03049] [INSPIRE].

[48] V. Cirigliano, W. Dekens, J. de Vries and E. Mereghetti, Constraining the top-Higgs sector of the Standard Model effective field theory, Phys. Rev. D 94 (2016) 034031 [arXiv: 1605. 04311] [INSPIRE].

[49] D. Barducci et al., Interpreting top-quark LHC measurements in the standard-model effective field theory, arXiv:1802.07237 [INSPIRE].

[50] G. Apollinari et al., High-Luminosity Large Hadron Collider (HL-LHC): preliminary design report, 10.5170/CERN-2015-005 (2015).

[51] CMS collaboration, Search for excited quarks of light and heavy flavor in $\gamma+$ jet final states in proton-proton collisions at $\sqrt{s}=13$ TeV, Phys. Lett. B 781 (2018) 390 [arXiv: 1711.04652] [INSPIRE].

[52] I.I.Y. Bigi et al., Production and decay properties of ultraheavy quarks, Phys. Lett. B 181 (1986) 157 [INSPIRE].

[53] M. Buchkremer and A. Schmidt, Long-lived heavy quarks: a review, Adv. High Energy Phys. 2013 (2013) 690254 [arXiv:1210.6369] [INSPIRE].

[54] L. Lee, C. Ohm, A. Soffer and T.-T. Yu, Collider searches for long-lived particles beyond the standard model, Prog. Part. Nucl. Phys. 106 (2019) 210 [arXiv:1810.12602] [INSPIRE].

[55] ATLAS collaboration, Search for heavy charged long-lived particles in the ATLAS detector in $36.1 \mathrm{fb}^{-1}$ of proton-proton collision data at $\sqrt{s}=13 \mathrm{TeV}$, Phys. Rev. D 99 (2019) 092007 [arXiv: 1902.01636] [INSPIRE].

[56] J.A. Aguilar-Saavedra and M. Pérez-Victoria, Top couplings and top partners, J. Phys. Conf. Ser. 452 (2013) 012037 [arXiv: 1302 .5634] [inSPIRE].

[57] ATLAS collaboration, Combination of the searches for pair-produced vector-like partners of the third-generation quarks at $\sqrt{s}=13$ TeV with the ATLAS detector, Phys. Rev. Lett. 121 (2018) 211801 [arXiv: 1808.02343] [INSPIRE].

[58] CMS collaboration, Search for pair production of vectorlike quarks in the fully hadronic final state, Phys. Rev. D 100 (2019) 072001 [arXiv:1906.11903] [INSPIRE].

[59] M. Chala, Direct bounds on heavy toplike quarks with standard and exotic decays, Phys. Rev. D 96 (2017) 015028 [arXiv: 1705.03013] [INSPIRE].

[60] H. Alhazmi, J.H. Kim, K. Kong and I.M. Lewis, Shedding light on top partner at the LHC, JHEP 01 (2019) 139 [arXiv: 1808. 03649] [INSPIRE].

[61] G.C. Branco and L. Lavoura, On the addition of vector like quarks to the Standard Model, Nucl. Phys. B 278 (1986) 738 [INSPIRE]. 
[62] J.A. Aguilar-Saavedra, Effects of mixing with quark singlets, Phys. Rev. D 67 (2003) 035003 [Erratum ibid. D 69 (2004) 099901] [hep-ph/0210112] [INSPIRE].

[63] A. Atre, G. Azuelos, M. Carena, T. Han, E. Ozcan, J. Santiago et al., Model-Independent Searches for New Quarks at the LHC, JHEP 08 (2011) 080 [arXiv:1102.1987] [INSPIRE].

[64] G. Cacciapaglia et al., Heavy vector-like top partners at the LHC and flavour constraints, JHEP 03 (2012) 070 [arXiv:1108.6329] [INSPIRE].

[65] R. Barcelo et al., Single vectorlike quark production at the LHC, Nucl. Phys. B 857 (2012) 172 [arXiv: 1110.5914] [INSPIRE].

[66] G. Cacciapaglia, T. Flacke, M. Park and M. Zhang, Exotic decays of top partners: mind the search gap, Phys. Lett. B 798 (2019) 135015 [arXiv:1908.07524] [INSPIRE].

[67] B.A. Dobrescu and F. Yu, Exotic signals of vectorlike quarks, J. Phys. G 45 (2018) 08LT01 [arXiv: 1612.01909] [INSPIRE].

[68] T.K. Hemmick et al., A search for anomalously heavy isotopes of low Z nuclei, Phys. Rev. D 41 (1990) 2074 [INSPIRE].

[69] R.N. Mohapatra and S. Nussinov, Possible manifestation of heavy stable colored particles in cosmology and cosmic rays, Phys. Rev. D 57 (1998) 1940 [hep-ph/9708497] [INSPIRE].

[70] R.N. Mohapatra and V.L. Teplitz, Primordial nucleosynthesis constraint on massive, stable, strongly interacting particles, Phys. Rev. Lett. 81 (1998) 3079 [hep-ph/9804420] [INSPIRE].

[71] M. Kusakabe, T. Kajino, T. Yoshida and G.J. Mathews, Effect of long-lived strongly interacting relic particles on Big Bang nucleosynthesis, Phys. Rev. D 80 (2009) 103501 [arXiv:0906.3516] [INSPIRE].

[72] D. Javorseket al., New experimental limits on strongly interacting massive particles at the TeV scale, Phys. Rev. Lett. 87 (2001) 231804 [InSPIRE].

[73] V. De Luca et al., Colored dark matter, Phys. Rev. D 97 (2018) 115024 [arXiv:1801.01135] [INSPIRE]. 